\title{
Evolution of Fusarium tricinctum and Fusarium avenaceum mitochondrial genomes is driven by mobility of introns and of a new type of palindromic microsatellite repeats
}

Nadia Ponts ${ }^{1}$, Charlotte Gautier ${ }^{1}$, Jérôme Gouzy ${ }^{2}$, Laetitia Pinson-Gadais' ${ }^{1}$, Marie Foulongne-Oriol ${ }^{1}$, Christine Ducos ${ }^{1}$, Florence Richard-Forget ${ }^{1}$, Jean-Michel Savoie ${ }^{1}$, Chen Zhao ${ }^{3}$ and Gérard Barroso ${ }^{1,4^{*}}$ (D)

\begin{abstract}
Background: Increased contamination of European and Asian wheat and barley crops with "emerging" mycotoxins such as enniatins or beauvericin, produced by Fusarium avenaceum and Fusarium tricinctum, suggest that these phylogenetically close species could be involved in future food-safety crises.

Results: The mitochondrial genomes of F. tricinctum strain INRA104 and F. avenaceum strain FaLH27 have been annotated. A comparative analysis was carried out then extended to a set of 25 wild strains. Results show that they constitute two distinct species, easily distinguished by their mitochondrial sequences. The mitochondrial genetic variability is mainly located within the intergenic regions. Marks of variations show they have evolved (i) by Single Nucleotide Polymorphisms (SNPs), (ii) by length variations mediated by insertion/deletion sequences (Indels), and (iii) by length mutations generated by DNA sliding events occurring in mononucleotide $(A)_{n}$ or $(T)_{n}$ microsatellite type sequences arranged in a peculiar palindromic organization. The optionality of these palindromes between both species argues for their mobility. The presence of Indels and SNPs in palindrome neighbouring regions suggests their involvement in these observed variations. Moreover, the intraspecific and interspecific variations in the presence/absence of group I introns suggest a high mobility, resulting from several events of gain and loss during short evolution periods. Phylogenetic analyses of intron orthologous sequences suggest that most introns could have originated from lateral transfers from phylogenetically close or distant species belonging to various Ascomycota genera and even to the Basidiomycota fungal division.

(Continued on next page)
\end{abstract}

* Correspondence: gerard.barroso@u-bordeaux.fr

'INRAE, MycSA, F-33882 Villenave d'Ornon, France

${ }^{4}$ University of Bordeaux, INRAE, MycSA, F-33882 Villenave d'Ornon, France

Full list of author information is available at the end of the article

(c) The Author(s). 2020 Open Access This article is licensed under a Creative Commons Attribution 4.0 International License, which permits use, sharing, adaptation, distribution and reproduction in any medium or format, as long as you give appropriate credit to the original author(s) and the source, provide a link to the Creative Commons licence, and indicate if changes were made. The images or other third party material in this article are included in the article's Creative Commons licence, unless indicated otherwise in a credit line to the material. If material is not included in the article's Creative Commons licence and your intended use is not permitted by statutory regulation or exceeds the permitted use, you will need to obtain permission directly from the copyright holder. To view a copy of this licence, visit http://creativecommons.org/licenses/by/4.0/ The Creative Commons Public Domain Dedication waiver (http://creativecommons.org/publicdomain/zero/1.0/) applies to the data made available in this article, unless otherwise stated in a credit line to the data. 


\begin{abstract}
(Continued from previous page)
Conclusions: Mitochondrial genome evolution between F. tricinctum and F. avenaceum is mostly driven by two types of mobile genetic elements, implicated in genome polymorphism. The first one is represented by group I introns. Indeed, both genomes harbour optional (inter- or intra-specifically) group I introns, all carrying putatively functional hegs, arguing for a high mobility of these introns during short evolution periods. The gain events were shown to involve, for most of them, lateral transfers between phylogenetically distant species. This study has also revealed a new type of mobile genetic element constituted by a palindromic arrangement of (A) $n$ and (T) $n$ microsatellite sequences whose presence was related to occurrence of SNPS and Indels in the neighbouring regions.
\end{abstract}

Keywords: Group I intron, Homing endonuclease, Lateral transfer, Palindrome, Fusarium tricinctum species complex

\section{Background}

Fusarium Head Blight (FHB) is a fungal disease of cereals caused by infection of grains by various (up to 15) Fusarium species [1]. Among these Fusarium species, five are commonly associated in cereal crops grown in Europe: Fusarium graminearum, Fusarium culmorum, Fusarium avenaceum, Fusarium tricinctum and Fusarium poae [2]. These five species are all able to produce mycotoxins that accumulate in grains and contaminate processed food and feed products, representing both a health risk and an important economic stake. Several of these mycotoxins (e.g., deoxynivalenol and zearalenone) are targeted by European and international regulations fixing maximum admissible levels in food and feeds (regulation EC 2006/2009). Others, such as fusaproliferin, moniliformin, enniatins and beauvericin, are increasingly found as crop contaminants and considered as "emerging" [3]. Recent toxicological studies suggested enniatins could be genotoxic and hepatotoxic [4], and could be involved in future food-safety crises, especially in a context of climate and agricultural practices changes. As an example, a three-year study (2011-2013) on 11 malting barley varieties cultivated in Italy revealed changes in the Fusarium species constituting the FHB complex depending on the variety used and/or specific weather parameters occurring during the seasons, the enniatin-producers $F$. avenaceum and Fusarium tricinctum being consistently present [1]. This study confirmed previous experiments of wheat ears co-inoculation by two different Fusarium species [5] which have shown that an increase of wetness period and temperature led to an increase of the FHB symptoms and of the mycotoxin (trichothecene) productivity (up to 1000 times). Similarly, numerous studies reported increased frequencies of contamination of European and Asian wheat and barley crops with enniatins mainly produced by $F$. avenaceum and $F$. tricinctum $[2,6,7]$. In a recent study, Orlando et al. [8] analyzed enniatin-contents and contaminations with Fusarium species in 1240 samples of small grain cereals (wheat, durum wheat, spring barley, triticale and winter barley) from 2012 to 2014 French harvests, and found enniatins produced by $F$. avenaceum and F. tricinctum highly prevalent in French small grain cereals at levels consistently at their highest on spring barley (mean values of 199 to $1316 \mu \mathrm{g} / \mathrm{kg}$ ).

In the past decade, phylogenetic studies as well as fungal genetic and genomic approaches have led to an important increase in the number of species identified as belonging to the Fusarium monophyletic genus [9]. Among them, F. avenaceum (Fries) [10] and F. tricinctum (Corda) [11] are considered as two closely related taxa ranged in the same $F$. tricinctum species complex [12]. In this Fusarium tricinctum species complex (FTSC), Fusarium acuminatum and Fusarium arthrosporioides $[13,14]$ appear also closely related to both species, whereas Fusarium torulosum, Fusarium flocciferum and Fusarium petersiae are grouped in a more distant sister clade $[12,15]$. In any case, the scarcity of data on both the genetic diversity of these fungal phytopathogens and the biosynthesis and regulation pathways of their associated mycotoxins limits our ability to assess the toxinogenic risk they represent, and consequently to design appropriate responses. In this context, the complete genome sequence of $F$. tricinctum strain INRA104 has recently been obtained in a whole genome shotgun project, including its full-length gapless mitochondrial genome [16]. In the present study, we investigated the modalities of molecular evolution of mitochondrial genomes in the Fusarium genus. We annotated the mitochondrial genome (MtDNA) of $F$. tricinctum strain INRA104 and compared its molecular organization with that of previously sequenced mitochondrial genomes of $F$. avenaceum [17] as well as of other distant Fusarium species $[18,19]$. Then, to study the phylogenetic relationship between these closely related species and assessing the accuracy of mitochondrial sequences to discriminate $F$. tricinctum and $F$. avenaceum, the polymorphic mitochondrial regions were characterized and their intra- and inter- specific variability was studied in a set of 25 previously assigned wild strains. The mitochondrial polymorphism was compared with that of two nuclear genes $r p b 1$ and $r p b 2$, previously reported as discriminating markers of both species [12]. The mobility and origin of all the harboured mitochondrial group I introns was also studied. 


\section{Results}

Molecular organisation and phylogenetic analysis of the mitochondrial genomes

From our whole genome sequencing project (DDB/ ENA/GenBank accession number QFZF00000000), the sequence of the complete mitochondrial genome (GenBank accession number CM009895) of F. tricinctum strain INRA104 has been retrieved and annotated (FtriMtDNA). In parallel, the mitochondrial genome of F. avenaceum strain FaLH27 (GenBank accession number JQGE01000002.1), a phylogenetically closely related species, was retrieved from third party sequencing data produced by Lysøe et al. [17] and also annotated for the purpose of comparison. The mitochondrial genomes of F. avenaceum strain FaLH27 (FaveMtDNA) and $F$. tricinctum strain INRA 104 have a size of $49,396 \mathrm{bp}$ and $48,506 \mathrm{bp}$, respectively and the same average GCcontent of $33 \%$. A second F. avenaceum annotated mitochondrial genome sequence was also found available in the GenBank for strain FaLH03 (49,402 bp, accession number JQGD01000004.1). It differs from FaveMtDNA strain FaLH27 by only $6 \mathrm{bp}$ in length, and possesses more than $99.9 \%$ of nucleotide identity. To avoid redundancy, the sequence of the MtDNA of the FaLH03 strain was not included in this study. F. tricinctum strain INRA104 and F. avenaceum strain FaLH27 MtDNAs are close in size, FaLH27 MtDNA being 890 bp longer (representing $1.8 \%$ of variation in length scattered along the whole mitochondrial genome), and show high sequence conservation with $98.9 \%$ nucleotide identity.

Annotation results of MtDNAs from both F. tricinctum strain INRA104 and F. avenaceum strain FaLH27 are displayed in Fig. 1, Additional file 1 (GenBank formatted annotated sequence of the $F$. tricinctum strain INRA104 MtDNA) and Additional file 2 (GenBank formatted annotated sequence of the $F$. avenaceum strain FaLH27 MtDNA). For easier comparison of the molecular organization of the F. tricinctum and F. avenaceum MtDNAs with others previously annotated MtDNAs in the fungal kingdom [20] and especially in the Fusarium genus, including those reported by Al-Reedy et al. [18], both sequences were annotated by arbitrarily fixing the first nucleotide as the $5^{\prime}$ end of the $r n l$ gene encoding the large ribosomal subunit RNA. This first nucleotide corresponds to positions $40,818 \mathrm{bp}$ in $F$. tricinctum INRA 104 MtDNA and $21,516 \mathrm{bp}$ in $F$. avenaceum FaLH27 one (GenBank accessions CM009895 and JQGE01000002, respectively).

F. tricinctum and F. avenaceum MtDNAs possess the same set of mitochondrial structural genes than all the Fusarium mitochondrial genomes reported to date [18]. Fourteen typical mitochondrial genes encode subunits of

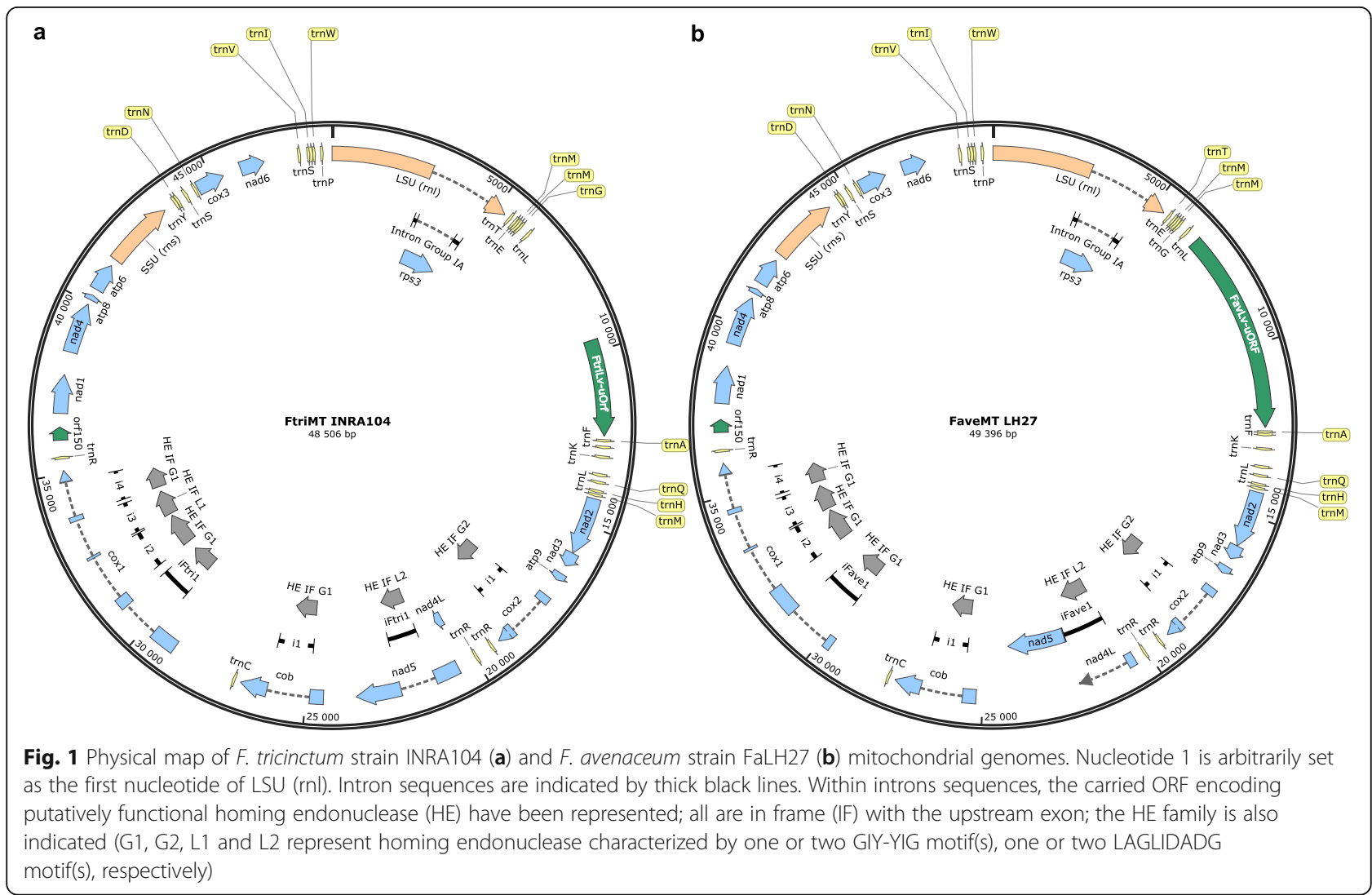


the electron transport chain and of the ATP-synthase complex. They include seven subunits of the electron transport complex I (nad1, 2, 3, 4, 4L, 5 and 6), one subunit of complex III ( $c o b)$, three subunits of complex IV (coxl, 2 and 3) and three subunits of the $\mathrm{F}_{0}$ ATP-synthase complex (atp6, 8 and 9). Fusarium mitochondrial coding sequences (CDS) show a conserved synteny (Fig. 2). All genes are indeed located on the same strand and share the same order. Notably, in all analysed Fusarium species, nad 2 and nad 3 genes are joined (no intergenic sequence), and nad4L and nad5 genes are fused, i.e., the last nucleotide of the nad4 termination codon TAA is also the first nucleotide of the nad5 ATG initiation codon (Fig. 1 and Additional files 1 and 2). Regarding structural RNA genes, $F$. tricinctum and $F$. avenaceum MtDNAs possess the LSU and SSU rDNAs and the same set of 26 tRNAs, strictly identical in sequences, including one tRNA "Sup" type (anticodon TCA) that reads the tryptophane (W) codon TGA. This is the only tRNA coding the amino-acid W carried by these genomes. Among the genes encoding mitochondrial proteins, it is to be noted that the gene rps3 encoding a protein involved in the assembly of the mitochondrial ribosome is located in the intron of LSU-rDNA, a feature shared by all Fusarium MtDNAs reported to date, and also by a large number of filamentous and yeast Ascomycota [20,21]. The only variability observed in synteny concerns the presence/absence of several tRNAs, or their relocation in other intergenic regions of the genome (Fig. 2). For example, additional tRNAs have been described in $F$. oxysporum variant 2 (tRNAs G2 and L3), $F$. verticillioides (tRNA R3), F. solani (tRNA M3), and F. graminearum (tRNA G2 and Y1). The tRNAs A or R2 are relocated in all displayed $F$. oxysporum variants, and the order of the tRNAs G and L1 is inverted in F. solani (versus the other Fusarium spp.). tRNA genes occupy seven intergenic regions of the $F$. tricinctum and $F$. avenaceum MtDNAs in which they are either alone, by pairs, or grouped by four to seven (Figs. 1 and 2).

Phylogenetic relationship was inferred from the 14 mitochondrial CDS sequences (all mitochondrial structural genes nad1 to 6, cob, cox1 to 3, atp6, 8 and 9), concatenated, between 11 Fusarium species belonging to five species complexes (FSSC, FSAMSC, FTSC, FOSC, FFSC), seven distant Ascomycota and one Basidiomycota (Fig. 3). The size of the compiled sequences varied from 11,721 bp (for the Basidiomycota Agaricus bisporus) to 14, 769 bp (for the Ascomycota Epichloe typhina); from 12, $878 \mathrm{bp}$ (F. mangiferae) and 13,144 bp (F. fujikuroi) for species of the Fusarium genus. This tree is fully congruent with those reported in previous phylogenetic studies based on the nuclear markers $r p b 1$ and $r p b 2$ [12]. In this mitochondrial CDS-based tree, the FTSC appears distant from the Fusarium sambucinum Species Complex (FSAMSC) as well as the related Fusarium oxysporum (FOSC) and Fusarium fujikuroi (FFSC) Species Complexes. As previously reported [12], the Fusarium solani Species Complex (FSSC) ranges in an outgroup position in regard of the other Fusarium species complexes. For comparison, a phylogenetic tree based on the cox1 CDS (from $1587 \mathrm{bp}$ to $1593 \mathrm{bp}$ in all the available Fusarium species) has also been built (Additional file 3: Fig. S1). This gene has been chosen because it is known to possess the highest number of group I introns in the fungal kingdom and in the Fusarium genus [22], and consequently can also be used to evidence conflict in phylogenetic trees based on orthologous intronic sequences of mitochondrial genes. Both trees based on the 14 compiled CDS and on the cox 1 CDS are fully congruent, showing the lack of gene conflict between cox 1 exonic sequences and all other mitochondrial CDS from a phylogenetic point of view.

\begin{tabular}{|c|c|c|c|c|c|c|c|c|c|c|c|c|c|c|c|c|c|c|c|c|c|c|c|c|c|c|c|}
\hline F. tricinctum ... & $r n l$ & rps3 & $\mid r n l$ & $\mathrm{~T}$ & $\mathrm{E}$ & M1 & M2 & & G & & L1 & & uORF & A & $\mathrm{F}$ & & $|\mathrm{K}|$ & & L2 & & Q & $\mathrm{H}$ & M3 & nad2 & nad3 & atp9 & $\cos 2$ \\
\hline F. avenaceum ... & $r n l$ & rps3 & $r n l$ & $\mathrm{~T}$ & $\mathrm{E}$ & M1 & M2 & & G & & L1 & & uORF & A & $\mathrm{F}$ & & $\mathrm{K}$ & & L2 & & Q & $\mathrm{H}$ & M3 & nad2 & nad3 & atp9 & $\cos 2$ \\
\hline F. oxysporum 1 ... & $r n l$ & rps3 & $r n l$ & $\mathrm{~T}$ & $\mathrm{E}$ & M1 & M2 & & G & & L1 & & & & $\mathrm{F}$ & & $\mathrm{K}$ & A L L & L2 & & Q & $\mathrm{H}$ & M3 & nad2 & nad3 & atp9 & $\cos 2$ \\
\hline F. oxysporum $2 \ldots$ & $r n l$ & rps3 & $r n l$ & $\mathrm{~T}$ & $E$ & M1 & M2 & A & G1 & G2 & L1 & & & & $F$ & & $\mathrm{~K}$ & & L2 & L3 & Q & $\mathrm{H}$ & M3 & nad2 & nad3 & atp9 & $\cos 2$ \\
\hline F. oxysporum $3 \ldots$ & $r n l$ & rps3 & $r n l$ & $\mathrm{~T}$ & E & M1 & M2 & & G & & L1 & & uORF & A & $\mathrm{F}$ & & $\mathrm{K}$ & & L2 & & $Q$ & $\mathrm{H}$ & M3 & nad2 & nad3 & atp9 & $\cos 2$ \\
\hline F. verticillioides & $r n l$ & rps3 & $r n l$ & $\mathrm{~T}$ & $\mathrm{E}$ & M1 & M2 & & G & & L1 & & uORF & A & $\mathrm{F}$ & & $\mathrm{K}$ & & L2 & & Q & $\mathrm{H}$ & M3 & nad2 & nad3 & atp9 & $\cos 2$ \\
\hline F. solani & $r n l$ & rps3 & $r n l$ & $\mathrm{~T}$ & $\mathrm{E}$ & M1 & M2 & & L1 & & G & & uORF & A & $\mathrm{F}$ & M3 & $\mathrm{K}$ & & L2 & & $Q$ & $\mathrm{H}$ & & nad2 & nad3 & atp9 & $\cos 2$ \\
\hline F. graminearum & $|r n l|$ & rps3 & $r n l$ & $\mathrm{~T}$ & E & M1 & M2 & & G1 & & L1 & G2 & uORF & A & $\mathrm{F}$ & & $|\mathrm{K}|$ & & L2 & & Q & $\mathrm{H}$ & M3 & nad2 & nad3 & atp9 & $\cos 2$ \\
\hline F. tricinctum & R1 & $\mathrm{R} 2$ & & nad4L & nad5 & & $c o b$ & C & $\operatorname{cox} 1$ & |R3 & nad1 & nad4 & atp8 & atp6 & rns & $Y$ & $\mathrm{D} / \mathrm{S}$ & S1 & $\mathrm{N}$ & $\cos 3$ & nad6 & $\mathrm{V} \mid$ & 1 & S2 & W & $P$ & $\ldots$ \\
\hline F. avenaceum & R1 & $\mathrm{R} 2$ & & ad $4 L$ & nad5 & & $c o b$ & C & $\cos 1$ & R3 & nad1 & nad4 & atp8 & atp6 & rns & $Y$ & $D S$ & S1 & $\mathrm{N}$ & $\cos 3$ & nad6 & $\mathrm{V}$ & 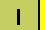 & S2 & W & $P$ & $\cdots$ \\
\hline F. oxysporum 1 & R1 & & & nad $4 L$ & nad5 & R2 & $c o b$ & C & $\operatorname{cox} 1$ & R3 & nad1 & nad4 & atp8 & atp6 & rns & $\mathrm{Y}$ & $\mathrm{D} S \mathrm{~S}$ & S1 & $\mathrm{N}$ & $\cos 3$ & nad6 & $\mathrm{V}$ & I & S2 & W & $\mathrm{P}$ & $\ldots$ \\
\hline F. oxysporum 2 & R1 & & & nad $4 L$ & nad5 & R2 & $c o b$ & C & $\cos 1$ & R3 & nad1 & nad4 & atp8 & atp6 & rns & $\mathrm{Y}$ & $D S$ & S1 & $\mathrm{N}$ & $\cos 3$ & nad6 & $\mathrm{V}$ & I & S2 & W & $\mathrm{P}$ & 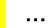 \\
\hline F. oxysporum 3 & R1 & & & nad4L & nad5 & R2 & $c o b$ & C & $\operatorname{cox} 1$ & R3 & nad1 & nad4 & atp8 & atp6 & rns & $\mathrm{Y}$ & $\mathrm{D} S \mathrm{~S}$ & S1 & $\mathrm{N}$ & $\cos 3$ & nad6 & $\mathrm{V}$ & I & S2 & W & $\mathrm{P}$ & .. \\
\hline F. verticillioides & R1 & R2 & & nad4L & nad5 & R3 & $c o b$ & C & $\cos 1$ & R4 & nad1 & nad4 & atp 8 & atp6 & rns & $\mathrm{Y}$ & $\mathrm{D} S \mathrm{~S}$ & S1 & $\mathrm{N}$ & $\cos 3$ & nad6 & $\mathrm{V}$ & t & S2 & W & $P$ & $\cdots$ \\
\hline F. solani & R1 & & & nad $4 L$ & nad5 & & $c o b$ & C & $\cos 1$ & R2 & nad1 & nad4 & atp8 & atp6 & rns & Y & $D$ & S1 & $\mathrm{N}$ & $\cos 3$ & nad6 & $\mathrm{V}$ & I & S2 & W & $\mathrm{P}$ & $\ldots$ \\
\hline F. graminearum & R1 & $\mathrm{R} 2$ & $\mathrm{Y} 1$ & nad4L & nad5 & & $c o b$ & $|\mathrm{C}|$ & $\cos 1$ & R3 & nad1 & nad4 & atp8 & atp6 & rns & Y2 & $\mathrm{D} / \mathrm{s}$ & S1 & $\mathrm{N}$ & $\cos 3$ & nad6 & $\mathrm{V} \mid$ & 1 & S2 & W & $\mathrm{P}$ & $\ldots$ \\
\hline
\end{tabular}

Fig. 2 Schematic representation of gene synteny in Fusarium mitochondrial genomes. Annotations for F. oxysporum, F. graminearum and F. solani were retrieved from Al-Reedy et al. [16] and Brankovics et al. [17]. F. oxysporum 1, 2 and 3 are the three variants of the large variable region characterized in Brankovics et al. [17], strains Fon015, FOSC3-a, and NRRL37622, respectively. Annotations for F. tricinctum strain INRA104 and F. avenaceum strain FaLH27 are from the present study. rDNA genes $r n /$ and $r n s$ are in light orange; typical mitochondrial protein genes are in pale blue; tRNAs in yellow to olive green shades; uORFs are in bright green 


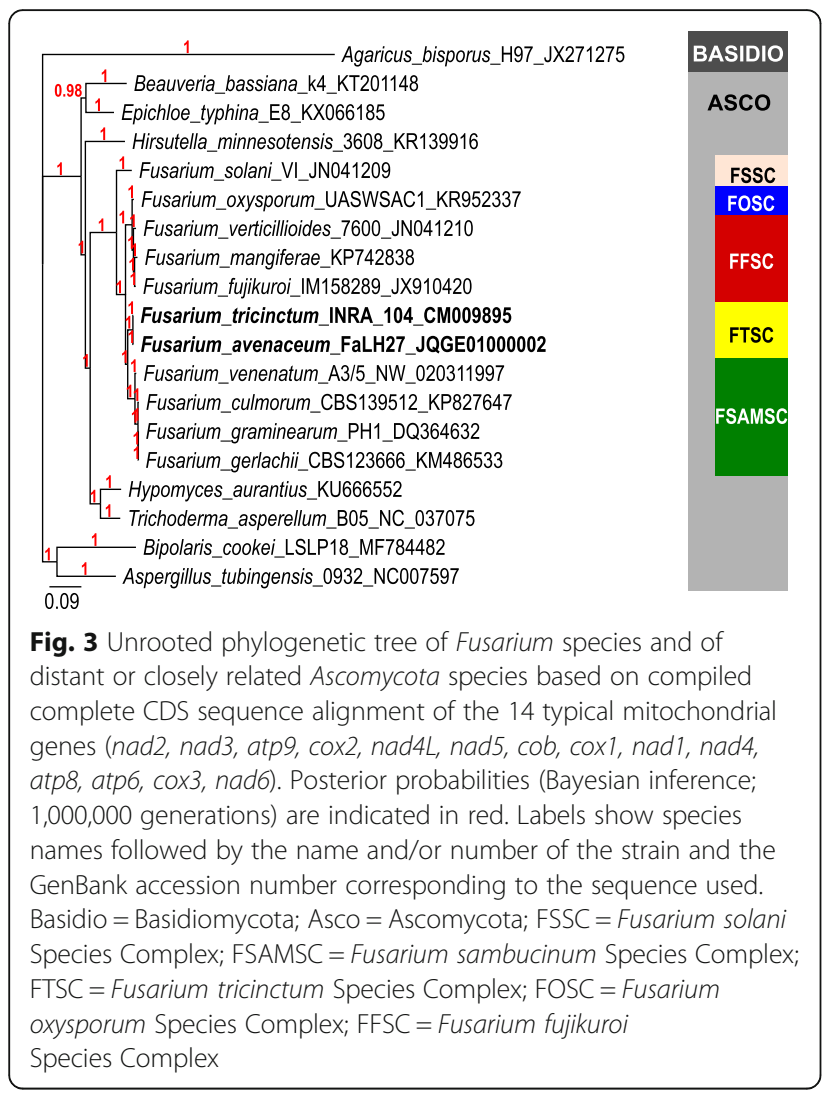

\section{Location of polymorphic versus conserved features}

The analysis of the overall molecular organisation of F. tricinctum and $F$. avenaceum MtDNAs allowed distinguishing two remarkable regions: $(i)$ a large region constituted by a long unidentified open reading frame flanked by two tRNAs clusters, previously named as Lv-uORF (for large variable unidentified ORF) in other Fusaria by Al-Reedy et al. [18]; (ii) the rest of the genome containing all conserved typical mitochondrial genes (39,586 bp and 40,439 bp in length in F. tricinctum and $F$. avenaceum, respectively). In $F$. avenaceum, the uORF included in this 8956 bp-long region (from nt 5290 to nt 14,245 ) is $5865 \mathrm{bp}$ in size (from nt 6503 to nt 12,367). This ORF is reduced in F. tricinctum strain INRA104 which harbours an eroded uORF of only $2748 \mathrm{bp}$ corresponding to the 3'end of the $F$. avenaceum one (for a total variable region of $8919 \mathrm{bp}$ in length in F. tricinctum, spanning from $5261 \mathrm{bp}$ to $14,179 \mathrm{bp}$ ).

CDS encoding mitochondrial proteins of both F. tricinctum and $F$. avenaceum were found highly conserved in sequence and length. For example, COX1 CDS sequences (1593 bp) of both species differ by only three SNPs $(99.8 \%$ of identity). When comparing with other species of the Fusarium genus, percentages of identity with F. tricinctum COX1 CDS varied between $92.5 \%$ for the distant $F$. solani, and $94-96 \%$ for members of the FOSC, FFSC or FSAMSC which includes $F$. graminearum.
As a whole, comparison of the mitochondrial genome of F. tricinctum (48,506 bp) with that of $F$. avenaceum (49, $396 \mathrm{bp})$ revealed a global mutation frequency of 7.83 events/kb explained by $314 \mathrm{SNPs}(6.47 \mathrm{SNP} / \mathrm{kb})$ and 66 Indels $(1.36$ Indel $/ \mathrm{kb})$. The total size of these Indel sequences represented $1713 \mathrm{bp}$, i.e., $3.5 \%$ of the $F$. tricinctum genome size (see Additional file 3: Table S1 and Table S2 for details). Although coding sequences (rDNA, tRNA, and protein-coding CDS) represent $43.8 \%$ of the total $F$. tricinctum mitochondrial genome, they contain less than $8 \%$ of all 380 mutations events (30 SNPs, or $1.41 \mathrm{SNP} / \mathrm{kb}$ ). With $1.63 \mathrm{SNP} / \mathrm{kb}$ and 2.21 Indels/kb, the introns found in these structural genes are only slightly more polymorphic, mostly due to the presence of Indels (absent from coding sequences). Most of the genetic variability (mainly composed of SNPs) is found in intergenic regions and in the large variable uORF-including region, which concentrate 47.9 and $39.2 \%$ of the mutation events in 22.1 and $16.4 \%$ of the genome, respectively (or 16.95 and 18.76 total mutation events/kb, respectively).

Strikingly, seven intergenic regions $\left(\mathrm{N}^{\circ} 18,19,20,23,25\right.$, 26 and 31) were shown to be affected by length variations occurring in microsatellites regions with a palindromic type organization (Fig. 4). Indeed, these regions are composed of (A) $)_{7-12}$-TATA (or TGTA or TACA or TA)-(T) ${ }_{9-11}$ sequences. Beside these polymorphic regions, this peculiar palindromic organization of mononucleotide microsatellites was found 14 and 16 times in F. avenaceum and F. tricinctum MtDNA sequences, respectively. In the details, palindromes were found in the polymorphic regions listed above, and also in both tRNAs clusters flanking the Lv-uORF as well as in the intergenic regions $\mathrm{N}^{\circ} 22$ and 28 (Fig. 4). When comparing both species, three palindromes were absent from FaveMtDNA (in the Lv-uORF and the intergenic regions 23 and 28) and one from FtriMtDNA (in the intergenic region 7). Moreover, these palindromes were frequently found associated with large deletions or sequences variations in the neighbouring regions (Fig. 4, lv-uORF, and intergenic regions $5,7,8,19,26$ and 28).

\section{Characterisation of mitochondrial introns and Iv-uORF of $F$. tricinctum strain INRA104 and $F$. avenaceum strain FaLH27}

MtDNAs of $F$. tricinctum strain INRA104 and $F$. avenaceum strain FaLH27 were shown to each harbour eight group I introns. Among them, the LSU-rDNA intron (group IA) containing the ribosomal protein rps3 gene is present in both $F$. tricinctum and F. avenaceum, as it is the case for all the mitochondrial genomes described to date in Fusarium species [18] and in most of the Ascomycota filamentous species [20, 21]. Regarding mitochondrial protein structural genes, five introns were present in both species: one in $\operatorname{cox} 2$ ( $\operatorname{cox} 2 \mathrm{i1})$, one in $c o b$ 


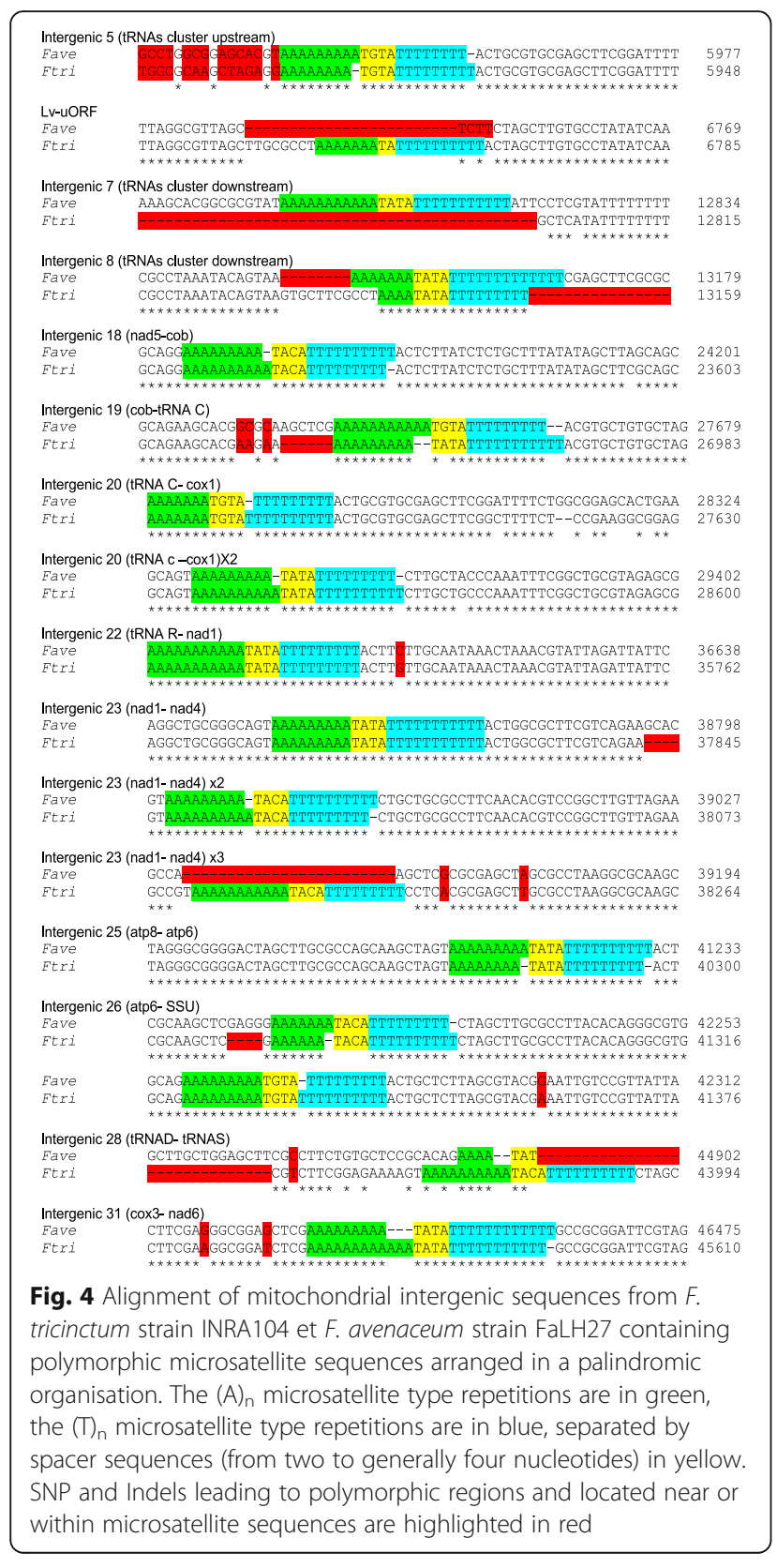

(cob i1), and three in $\operatorname{cox} 1$ ( $\operatorname{cox} 1 \mathrm{i2}$, $\operatorname{cox} 1 i 3$ and $\operatorname{cox} 1 \mathrm{i4}$ ). Each of these five introns was assumed to represent orthologous sequences on the basis of a shared insertion site in the CDS of the gene as well as a high nucleotide sequence identity (>99\%). In addition to these five conserved introns, $F$. tricinctum possesses one intron in nad5 (nad5 iFtri1) and one intron in the $5^{\prime}$ part of cox1 (coxl iFtri1) that are not present in F. avenaceum; in the same way, $F$. avenaceum MtDNA harbours an intron in nad4L (nad4L iFave1) that is not present in F. tricinctum as well as an intron located in the $5^{\prime}$ part of cox 1 (cox1 iFave1) but different from cox 1 iFtri1 in sequence (only $49.3 \%$ of nucleotide sequence identity) and in its insertion site. All these group I introns have a size between $1018 \mathrm{bp}$ (cox1 i4) and $1481 \mathrm{bp}$ (nad4L iFave1), and all carry an intact ORF in frame with the upstream exon which encodes a potentially functional homing endonuclease (HE) acting in the homing (site-specific integration) of the intron in the corresponding CDS after lateral transfer [23].

For each of these nine group I introns, their optionality (presence/absence pattern) was investigated in a panel of 25 strains constituted by $14 F$. tricinctum and $11 \mathrm{~F}$. avenaceum strains (Table 1 and Additional file 3: Table S3) by a fragment-length PCR approach that uses primers defined in exons flanking the investigated intron (Additional file 3: Table S4). Results, expressed by the presence or absence of each intron, were obtained for the $14 \mathrm{~F}$. tricinctum and $11 \mathrm{~F}$. avenaceum studied strains and compiled in Table 1. Among the five introns shared by $F$. tricinctum strain INRA104 and $F$. avenaceum strain FaLH27 - i.e., cox 2 il, cob i1, cox 1 i2, cox 1 i3, and cox1 it - the three introns located in the 3' part of the $\operatorname{cox} 1$ gene ( $\operatorname{cox} 1 i 2, i 3$ and $i 4$ ) were the only ones present in all the 25 studied strains. The intron cox 2 i1 was found in all but one strain, $F$. avenaceum INRA612. The intron cob i1 was missing in 9 out of 14 of the F. tricinctum strains. Considering the two introns found in $F$. tricinctum INRA104 and not in $F$. avenaceum FaLH27 i.e., cox1 iFtri1 and nad5 iFtri1 - cox1 iFtri1 was indeed present in all investigated $F$. tricinctum strains while absent from all $F$. avenaceum ones whereas nad5 iFtri1 was not exclusive to $F$. tricinctum but also found in two out of $11 F$. avenaceum strains ( $F$. avenaceum INRA6 and $F$. avenaceum INRA612). Sequencing of corresponding PCR products showed high nucleotide identity ( $99.5 \%$, i.e., five $\mathrm{SNP}$ on the whole $1.025 \mathrm{~kb}-$ long intron sequence). Regarding the two introns found in $F$. avenaceum FaLH27 but not in F. tricinctum INRA104 - i.e., nad4L iFave1 and cox1 iFave1, nad4L iFave1 was found in all $F$. avenaceum strains but not in any of the investigated $F$. tricinctum strains, whereas cox 1 iFave1 was present in all $F$. avenaceum strains but also in one F. tricinctum strain (INRA86). As a whole, 9 out of 11 of the $F$. avenaceum studied strains have identical intronic pattern. Two strains, $F$. avenaceum INRA6 and $F$. avenaceum INRA612, nonetheless differ by the presence of the nad5 iFtri1, and the lack of cox $2 i 1$ for strain INRA612. In the 14 F. tricinctum studied strains, two groups can be distinguished based on the absence or presence of $c o b i 1$, herein referred to as group A (nine strains) and group B (five strains), respectively. In group B, only strain INRA86 shows a specific intronic profile characterized by the additional presence of cox 1 iFave1.

For comparison purposes, we categorize the 25 strains of this study using as marker the partial sequences of PCR products derived from $r p b 1$ (798 bp), rpb2 (762 bp) 
Table 1 Optionality of F. tricinctum and F. avenaceum mitochondrial introns

\begin{tabular}{|c|c|c|c|c|c|c|c|c|c|c|}
\hline Species & Strain & $\cos 2 i 1$ & nad4L iFavel & nad5 iFtri1 & cob il & cox1 ifavel & cox1 iFtri1 & $\operatorname{cox} 1$ i2 & $\operatorname{cox} 1$ is & coxt is \\
\hline F. tricinctum & INRA104 & $P^{a}$ & Exon & $P$ & $P$ & Exon & $P$ & $P$ & $P$ & $P$ \\
\hline F. tricinctum & INRA 105 & $\mathbf{P}$ & Exon & $\mathbf{P}$ & $\mathbf{P}$ & Exon & $\mathbf{P}$ & $\mathbf{P}$ & $\mathbf{P}$ & $\mathbf{P}$ \\
\hline F. tricinctum & INRA 106 & $\mathbf{P}$ & Exon & $\mathbf{P}$ & $\mathbf{P}$ & Exon & $\mathbf{P}$ & $\mathbf{P}$ & $\mathbf{P}$ & $\mathbf{P}$ \\
\hline F. tricinctum & INRA 610 & $\mathbf{P}$ & Exon & $\mathbf{P}$ & $\mathbf{P}$ & Exon & $\mathbf{P}$ & $\mathbf{P}$ & $\mathbf{P}$ & $\mathbf{P}$ \\
\hline F. tricinctum & INRA 521 & $\mathbf{P}$ & Exon & $\mathbf{P}$ & Exon & Exon & $\mathbf{P}$ & $\mathbf{P}$ & $\mathbf{P}$ & $\mathbf{P}$ \\
\hline F. tricinctum & INRA 522 & $\mathbf{P}$ & Exon & $\mathbf{P}$ & Exon & Exon & $\mathbf{P}$ & $\mathbf{P}$ & $\mathbf{P}$ & $\mathbf{P}$ \\
\hline F. tricinctum & INRA 523 & $\mathbf{P}$ & Exon & $\mathbf{P}$ & Exon & Exon & $P$ & $\mathbf{P}$ & $\mathbf{P}$ & $\mathbf{P}$ \\
\hline F. tricinctum & INRA 524 & $\mathbf{P}$ & Exon & $\mathbf{P}$ & Exon & Exon & $\mathbf{P}$ & $\mathbf{P}$ & $\mathbf{P}$ & $\mathbf{P}$ \\
\hline F. tricinctum & INRA 525 & $\mathbf{P}$ & Exon & $\mathbf{P}$ & Exon & Exon & $\mathbf{P}$ & $\mathbf{P}$ & $\mathbf{P}$ & $\mathbf{P}$ \\
\hline F. tricinctum & INRA 526 & $\mathbf{P}$ & Exon & $\mathbf{P}$ & Exon & Exon & $\mathbf{P}$ & $\mathbf{P}$ & $\mathbf{P}$ & $\mathbf{P}$ \\
\hline F. tricinctum & INRA 527 & $\mathbf{P}$ & Exon & $\mathbf{P}$ & Exon & Exon & $\mathbf{P}$ & $\mathbf{P}$ & $\mathbf{P}$ & $\mathbf{P}$ \\
\hline F. tricinctum & INRA 528 & $\mathbf{P}$ & Exon & $\mathbf{P}$ & Exon & Exon & $\mathbf{P}$ & $\mathbf{P}$ & $\mathbf{P}$ & $\mathbf{P}$ \\
\hline F. tricinctum & INRA 529 & $\mathbf{P}$ & Exon & $\mathbf{P}$ & Exon & Exon & $\mathbf{P}$ & $\mathbf{P}$ & $\mathbf{P}$ & $\mathbf{P}$ \\
\hline F. tricinctum & INRA 86 & $\mathbf{P}$ & Exon & $\mathbf{P}$ & $\mathbf{P}$ & $\mathbf{P}$ & $\mathbf{P}$ & $\mathbf{P}$ & $\mathbf{P}$ & $\mathbf{P}$ \\
\hline F. avenaceum & $\mathrm{FaLH} 27$ & $\mathbf{P}$ & $\mathbf{P}$ & Exon & $\mathbf{P}$ & $\mathbf{P}$ & Exon & $\mathbf{P}$ & $\mathbf{P}$ & $\mathbf{P}$ \\
\hline F. avenaceum & INRA 112 & $\mathbf{P}$ & $\mathbf{P}$ & Exon & $\mathbf{P}$ & $\mathbf{P}$ & Exon & $\mathbf{P}$ & $\mathbf{P}$ & $\mathbf{P}$ \\
\hline F. avenaceum & INRA 494 & $\mathbf{P}$ & $\mathbf{P}$ & Exon & $\mathbf{P}$ & $\mathbf{P}$ & Exon & $\mathbf{P}$ & $\mathbf{P}$ & $\mathbf{P}$ \\
\hline F. avenaceum & INRA 495 & $\mathbf{P}$ & $\mathbf{P}$ & Exon & $\mathbf{P}$ & $\mathbf{P}$ & Exon & $\mathbf{P}$ & $\mathbf{P}$ & $\mathbf{P}$ \\
\hline F. avenaceum & INRA 496 & $\mathbf{P}$ & $\mathbf{P}$ & Exon & $\mathbf{P}$ & $\mathbf{P}$ & Exon & $\mathbf{P}$ & $\mathbf{P}$ & $\mathbf{P}$ \\
\hline F. avenaceum & INRA 497 & $\mathbf{P}$ & $P$ & Exon & $\mathbf{P}$ & $\mathbf{P}$ & Exon & $\mathbf{P}$ & $\mathbf{P}$ & $\mathbf{P}$ \\
\hline F. avenaceum & INRA 498 & $\mathbf{P}$ & $\mathbf{P}$ & Exon & $\mathbf{P}$ & $\mathbf{P}$ & Exon & $\mathbf{P}$ & $\mathbf{P}$ & $\mathbf{P}$ \\
\hline F. avenaceum & INRA 499 & $\mathbf{P}$ & $P$ & Exon & $\mathbf{P}$ & $\mathbf{P}$ & Exon & $\mathbf{P}$ & $\mathbf{P}$ & $\mathbf{P}$ \\
\hline F. avenaceum & INRA 611 & $\mathbf{P}$ & $\mathbf{P}$ & Exon & $\mathbf{P}$ & $\mathbf{P}$ & Exon & $\mathbf{P}$ & $\mathbf{P}$ & $\mathbf{P}$ \\
\hline F. avenaceum & INRA 6 & $\mathbf{P}$ & $\mathbf{P}$ & $\mathbf{P}$ & $\mathbf{P}$ & $\mathbf{P}$ & Exon & $\mathbf{P}$ & $\mathbf{P}$ & $\mathbf{P}$ \\
\hline F. avenaceum & INRA 612 & Exon & $\mathbf{P}$ & $\mathbf{P}$ & $\mathbf{P}$ & $\mathbf{P}$ & Exon & $\mathbf{P}$ & $\mathbf{P}$ & $\mathbf{P}$ \\
\hline
\end{tabular}

${ }^{\text {aPresent }}$

and a part of the mitochondrial Lv-uORF (818 bp) (Fig. 5a, $\mathrm{b}$ and $\mathrm{c}$, respectively). For each marker, all orthologous sequences of strains and species belonging to the FTSC and available in the GenBank (see Additional file 4 Table S6 for accession numbers) have been added to the analyses (i.e., 40 rpb1 sequences and $60 r p b 2$ sequences in total; see Fig. $5 \mathrm{a}$ and $\mathrm{b}$ ). The trees obtained with nuclear sequences are highly congruent, showing that $F$. tricinctum and $F$. avenaceum strains separate well from each other as well as from the other species included in this analysis, with the exception of $F$. arthrosporioides NRRL26416 that clusters with $F$. avenaceum strains. All other species were separated in a phylogenetically distant clade. Regarding F. acuminatum, the studied strains seem to cluster in a sister group of the F. tricinctum clade except for F. acuminatum NRRL28652 and NRRL28449 that regroup with $F$. avenaceum strains. These results suggest that $F$. acuminatum may be more closely related to $F$. tricinctum than $F$. avenaceum, strains NRRL28652 and NRRL28449 being exceptions possibly belonging to the $F$. avenaceum or a new undescribed species. Strikingly, the two clades separated on the base of the presence/absence of cob i1 described earlier, match the clear separation of $F$. tricinctum strains in two groups in the rpb1 and rpb2based trees (Fig. $5 a$ and b, respectively).

A similar analysis was performed using the mitochondrial sequences of the large variable intergenic region carrying the uORF only (Fig. 5c). The obtained tree clearly separated $F$. tricinctum and $F$. avenaceum into two clades but fails to discriminate most strains of the same species. Indeed, nucleotide identities vary from 99.9 to $100 \%$ between strains of the same species, and less than $97.6 \%$ between strains of both species. On the sequence alignment (Additional file 3: Fig. S2), two insertion/deletion events can be observed: a deletion of three nucleotides in F. tricinctum vs. $F$. avenaceum at the beginning of the alignment, and an insertion of 39 to 45 nucleotides. These events are responsible for the erosion of the uORF in the $F$. tricinctum strains. These Indels are accompanied by several flanking interspecific SNPs. Strikingly, the large F. tricinctum inserted sequence is bordered by a copy of a polymorphic 


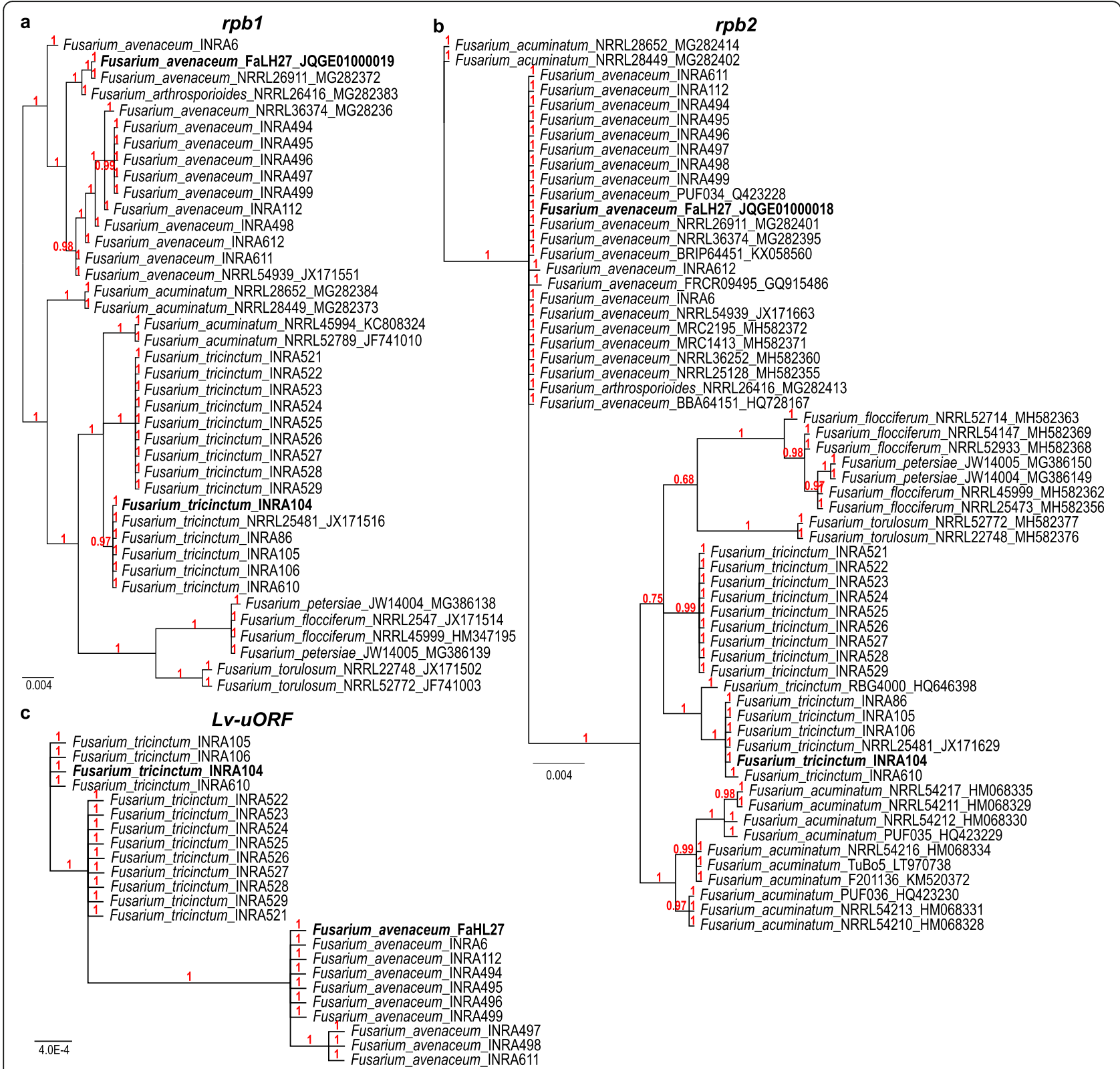

Fig. 5 Unrooted phylogenetic trees of strains belonging to the Fusarium tricinctum species complex (FTSC), including strains of the species $F$. tricinctum, F. avenaceum, F. acuminatum, F. arthrosporioides, F. torulosun, F. flocciferum, and F. petersiae. Trees are based on the nuclear markers rpb1 partial sequences (798 bp) (a), rpb2 partial sequence (762 bp) (b) or a part of the mitochondrial Lv-uORF (818 bp) (c). Posterior probabilities (Bayesian inference; 1,000,000 generations) are indicated in red. Species name is followed by the name of the strain and accession numbers when sequences were retrieved from Genbank

microsatellite region with the peculiar organization described earlier, i.e., $(\mathrm{A})_{7}-\mathrm{TA}-(\mathrm{T})_{9}-10$.

\section{Phylogenetic analyses of $F$. tricinctum and $F$. avenaceum mitochondrial group I introns}

To explore the origin and evolution history of each of these mitochondrial introns, orthologous sequences available in the GenBank have been compiled (see Additional file 4 Table S6 for accession numbers). Intron sequences were considered orthologous when they were members of the same Position class ( $\mathrm{PCl})$, as previously defined [22]: each $\mathrm{PCl}$ contains intron sequences possessing both the highest percentage of nucleotide identity and the same location in the exonic sequence (CDS). Results are summarized in Table 2.

Among the nine introns reported in F. tricinctum and $F$. avenaceum mitochondrial genes, only nad5 iFtri1 did not possess any orthologous sequences in other Fusarium 


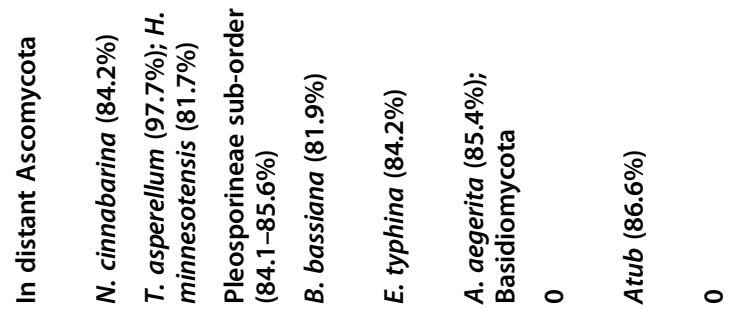

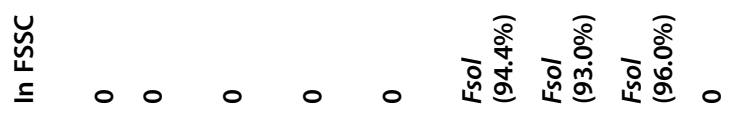
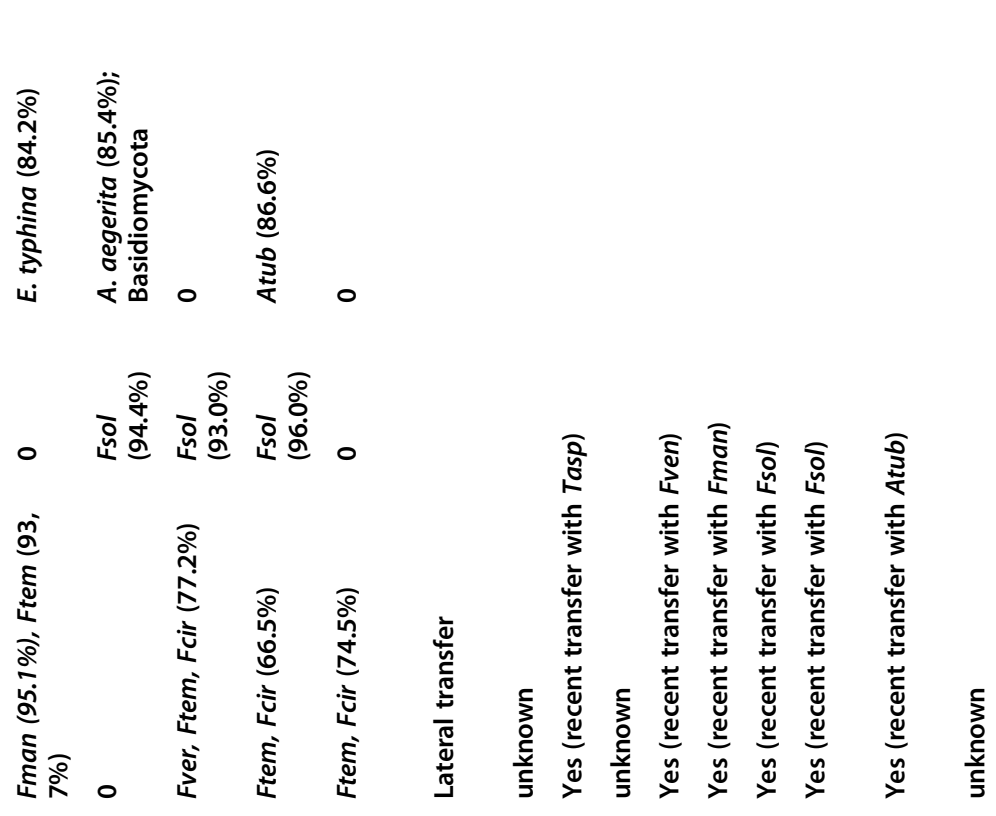

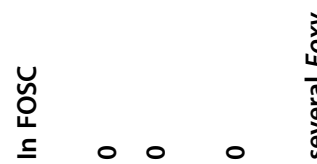

离

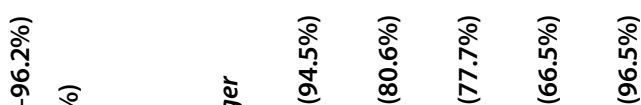

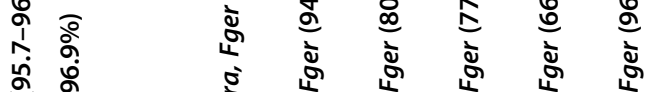

के

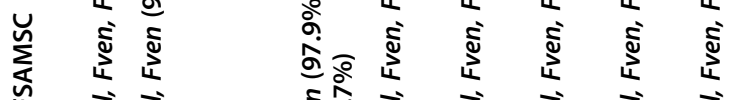

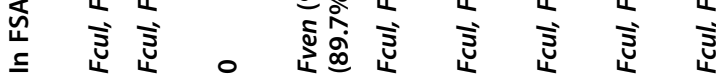

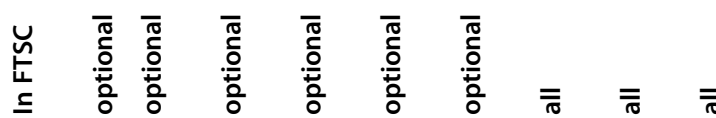

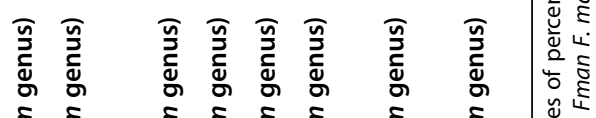
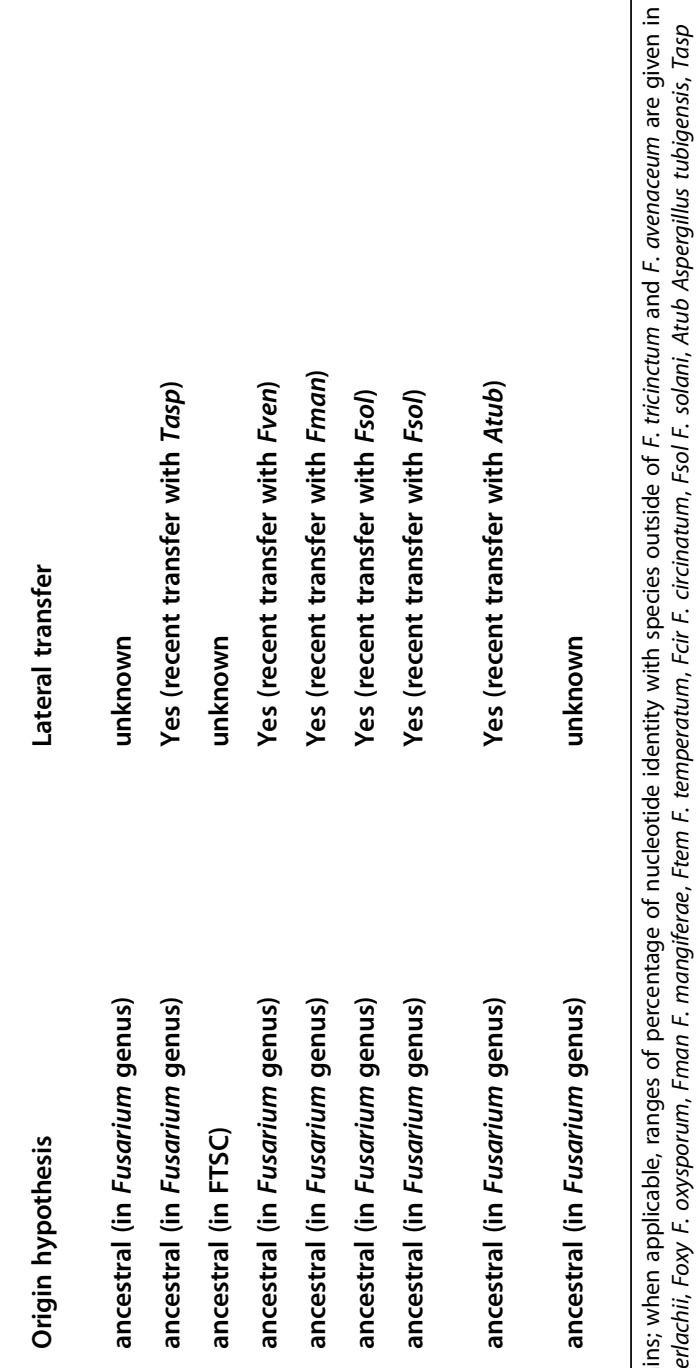

ते

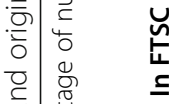

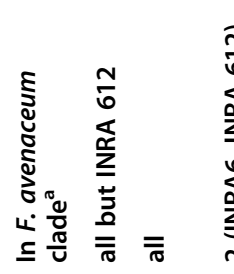

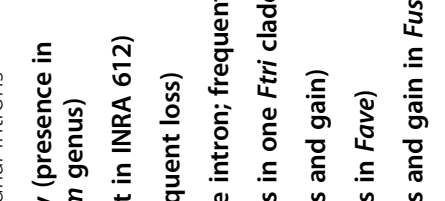

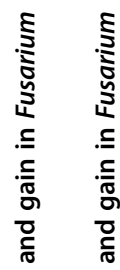

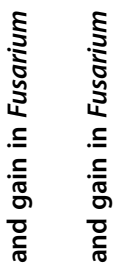

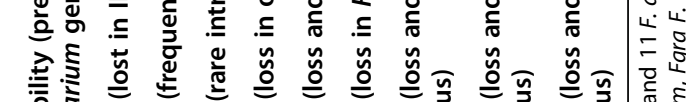

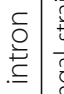

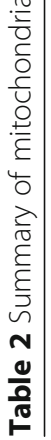

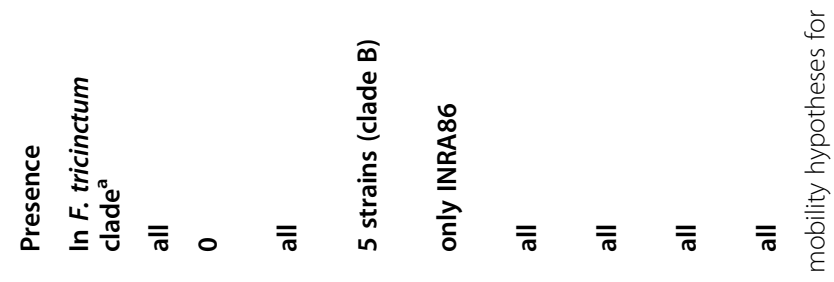

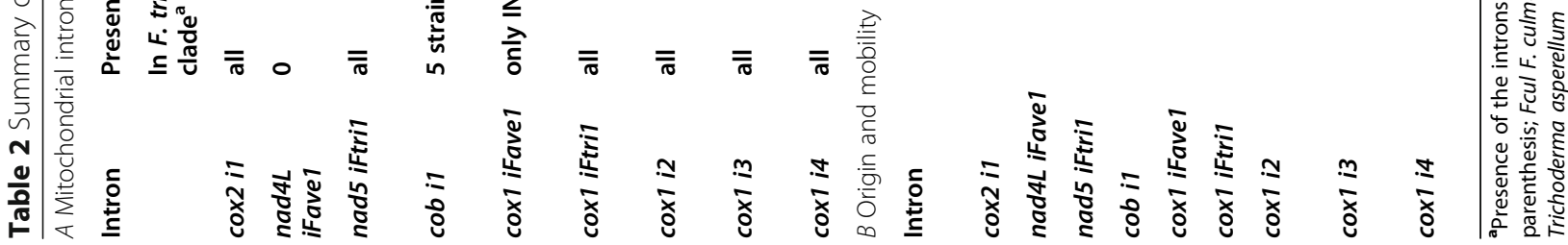


species. For this intron, only few orthologous sequences described in five Ascomycota species were found, all belonging to the Pleosporinae suborder, with 84.1 to $85.6 \%$ nucleotide identity: Bipolaris cookei and Bipolaris maydis, Leptosphaeria biglobosa 'brassicae', Leptosphaeria maculans 'lepidii', and Coniothyrium glycines.

Orthologous sequences of the eight remaining introns were found in other Fusarium species complexes, mainly in members of the FSAMSC. The cob i1 intron was shown to also possess orthologous sequences in members of the FOSC, whereas the five introns carried by $\operatorname{cox} 1$ had orthologous sequences in members of the FFSC with the exception of cox 1 iFtri1. Orthologous sequences of cox 1 iFtri1 as well as $\operatorname{cox} 1 i 2$ and $\operatorname{cox} 1 i 3$ were found in members of the FSSC. Notably, percentages of nucleotide identity between the various orthologous sequences included in this study were highly variable, arguing for different origins and evolution of the intronic sequences.

Phylogenetic trees were built based on the alignments of the orthologous sequences of each intron (Fig. 6). Whilst species of the FSAMSC, FTSC, and FFSC are well separated from each other and from other Ascomycota in the trees built from cox $i 1$ and $\operatorname{cox} 1$ i4 orthologous sequences (Fig. 6a and h, respectively), the Ascomycota Trichoderma asperellum is inserted among Fusarium species of the FSAMSC when nad4L iFave1 is considered (Fig. 6b), with 97\% nucleotide identity, suggesting a lateral transfer event. Similarly, at least five other lateral transfer events can be observed regarding cob1 i1 (involving Fusarium venenatum, Fig. 6c), cox1 iFave1 (involving Fusarium mangiferae, Fig. 6d), cox1 iFtri1 (involving the distant Basidiomycota Agrocybe aegerita, Fig. 6e), cox i3 (involving Aspergillus tubingensis, Fig. 6g), as well as three introns of $\operatorname{cox} 1$ ( $\operatorname{cox} 1$ iFtri1, cox1 $i 2$ and cox 1 i3) and involving F. solani (Fig. 6e, $\mathrm{f}$ and $\mathrm{g})$.

\section{Discussion}

The mitochondrial genomes of $F$. tricinctum strain INRA104 $(48.5 \mathrm{~kb})$ and $F$. avenaceum strain FaLH27 $(49.4 \mathrm{~kb})$ were found highly similar in size and sequence

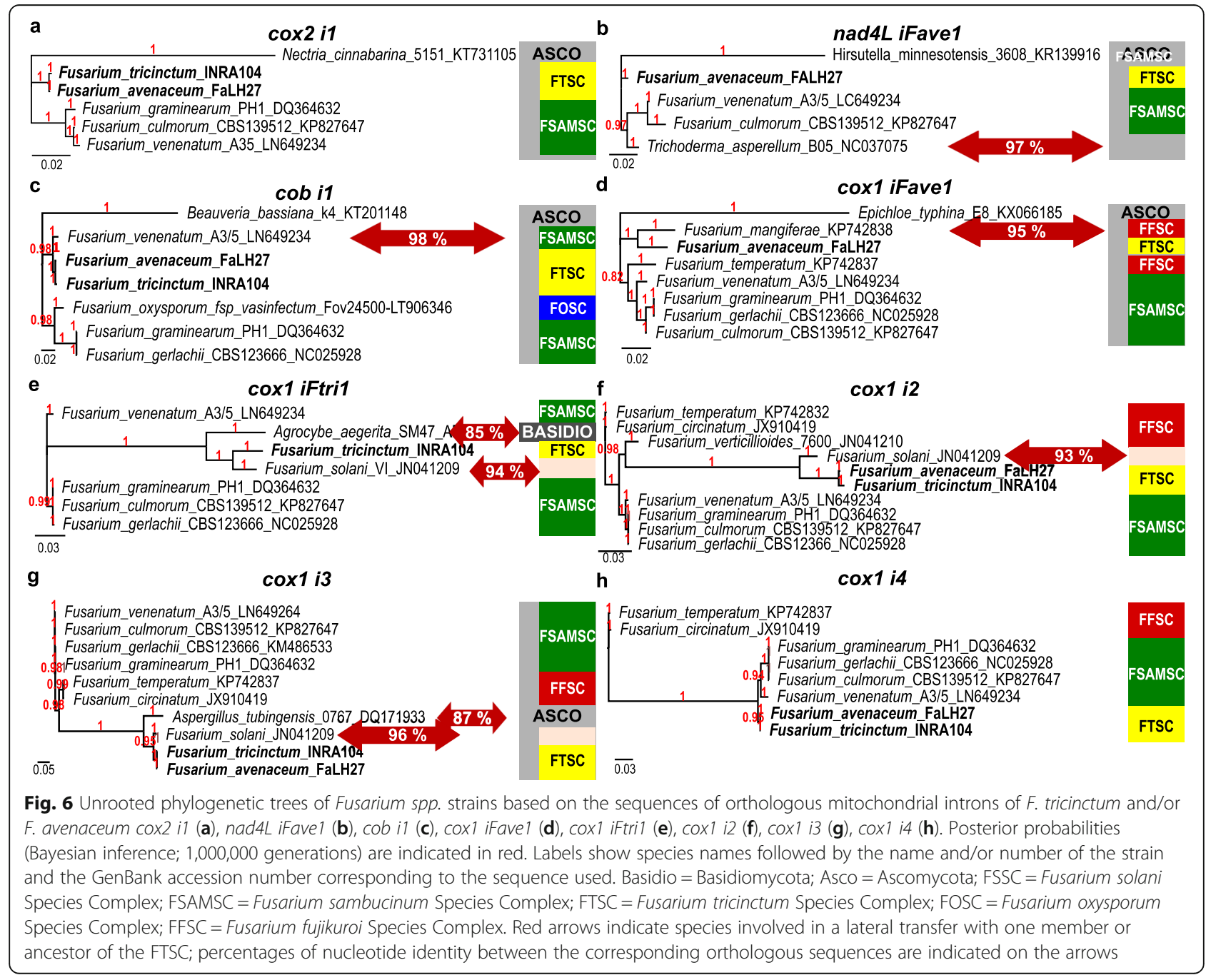


(98.3\% nucleotide identity), with an identical GC content (33\%). The comparative analysis of their molecular organization identified a variable region with high interspecific polymorphism, comprising an ORF with cryptic function (uORF of $5865 \mathrm{nt}, 1955 \mathrm{aa}$ ) flanked by two clusters of tRNAs, the ORF being eroded (2748 nt, $916 \mathrm{aa})$ in F. tricinctum strain INRA104 by comparison with the very large one found in F. avenaceum FaLH27. This region was previously defined by Al-Reedy et al. [18] as an "exceptionally large ORF" in F. verticillioides (2572 aa), F. solani (2013 aa) and F. graminearum (1931 aa). In a recent report based on the analysis of 61 mitochondrial genomes of the FOSC, Brankovics et al. [19] described a large uORF of 2284 aa in some strains or eroded smaller ORFs (less than $740 \mathrm{aa}$ ) in other strains, similarly to our observations in F. tricinctum and F. avenaceum. Here, we found that both the large variable uORF-including regions and intergenic regions exhibit similar patterns of frequent mutation events, thus arguing for the optionality of this large uORF. Searching nuclear genomes of uORF-devoid species/strains for the presence of paralogous sequences may provide elements towards understanding the evolution history of this optional ORF of cryptic function.

Surprisingly, we found that Lv-uORFs identified in F. tricinctum strain INRA104 and F. avenaceum FaLH27 as well as their flanking tRNAs clusters (in the intergenic regions 5 and 7) harboured microsatellite sequences $(\mathrm{A})_{\mathrm{n}}$ and $(\mathrm{T})_{\mathrm{n}}$ arranged in a palindrome-type organisation, and were also affected by large Indels and/or important sequence variations. Such association between this peculiar palindromic organisation and Indels was also found in several other intergenic regions of $F$. tricinctum and $F$. avenaceum mitochondrial genomes. The optionality of these palindromes between $F$. tricinctum and $F$. avenaceum argues for their mobility, as previously described for mitochondrial doublehairpin elements of Allomyces fungi [24]. Moreover, the frequent presence of large Indels and/or important sequence variations in the neighbouring regions of the palindromes of F. tricinctum and F. avenaceum suggests the involvement of this new type of putative mobile genetic elements in the observed deletions, and sequence evolution. Such repetitive palindromic repeats were found up to 19 times in the genome of the green microalga Lobosphaera incisa [25] and have been proposed to act as substrates for large-scale genomic rearrangements or as sites of transcript processing. In the same way, hairpin and tandem repeat-containing genetic elements, and also tRNAs with associated palindromic sequences have been described in cryptophyte algae, which possess a clear phylogenetic relationship to heterotrophic eukaryotes [26]. In these algae species, these sequences are believed to be involved in the observed gene shuffling affecting mitochondrial genes by promoting recombination events.
In fungal MtDNAs, dispersed repeated sequences have already been reported in filamentous or yeast Ascomycota, such as Pst1 (GC-rich) palindromes in Neurospora crassa $[27,28]$, the mitochondrial ultra-short element MUSE1 (11 bp in size, more than 100 copies per genome) in Podospora anserina [29], or GC clusters in Saccharomyces cerevisiae and 28 other yeast species [30]. Various roles have been proposed for such fungal interspersed elements, such as sites of initiation for replication or transcription, or processing of transcripts, but most of them are considered as mobile genetic elements, invasive and highly recombinogenic, and involved in mitochondrial polymorphism by generation of length mutations (excision, insertion, DNA slippage) [27-30].

To the best of our knowledge, the palindromic organisation of (A) n and (T) n microsatellites sequences described in the $F$. tricinctum and $F$. avenaceum MtDNA constitute the first report of such putatively mobile (ATrich) genetic elements. To date, nothing is known about their putative role (e.g., in the molecular evolution of mitochondrial genomes, the MtDNA replication or the regulation of the expression of genes). However, our results by showing a frequent link between these palindromes and the occurrence of SNP and/or Indels in their neighbouring regions (see Fig. 4), suggest a putative role of these mobile elements in the generation of mitochondrial polymorphism. Notably, despite the high number (14 to 16) of these palindromic sequences in FtriMtDNA and FaveMtDNA, no evidence of gene shuffling or recombination event was recovered.

When compared with other mitochondrial genomes available in the whole monophyletic genus Fusarium, the sizes of $F$. tricinctum and $F$. avenaceum MtDNAs are close to the sizes reported for 61 strains of the FOSC, ranging between $38.7 \mathrm{~kb}$ in $F$. oxysporum $f$. sp. melonis and $53.4 \mathrm{~kb}$ in $F$. oxysporum $f$. sp. lycopersici [19], as well as for F. verticillioides (53.7 kb) of the FFSC [18]. Other species have larger MtDNAs, such as $F$. solani $(63 \mathrm{~kb},[18])$ a member of the FSSC located at the base of the phylogenetic tree of the Fusarium genus [12]. Species of the FSAMSC have considerably larger MtDNAs [31, 32], in the range of 93.4 kp for Fusarium gerlachii (GenBank accession number KM486533) to $103.8 \mathrm{~kb}$ for F. culmorum (GenBank Accession number KP827647). As previously reported in numerous fungal genera [22], the mitochondrial genome size variations observed in the Fusarium genus does not involve the highly conserved set of mitochondrial genes and is not related to the phylogenetic distances separating these Fusarium species. Here, size variation would be mainly due to variations in the group I intron content of these genomes. For a still unknown reason, some species seem to behave as large intron reservoirs whereas other closely related ones show few or none introns [22]. 
Group I introns are mobile genetic elements that can be gained (after lateral transfers, even between phylogenetically distant species) or lost, during short evolution periods [22, 33]. The identification of putatively functional Homing Endonucleases genes (hegs) in all introns of F. tricintum and $F$. avenaceum mitochondrial genomes supports that the concerned introns are independent functional mobile genetic elements with homing specificity. Comparing the molecular organization of MtDNAs of F. tricinctum and F. avenaceum, we found significant polymorphisms between these closely related species in relation with intron mobility. This inter- or intra-specific optionality can be explained by two divergent hypotheses [22, 33-35]: (i) a gain of the mobile genetic element after a lateral transfer between a phylogenetically close or distant species $[35,36]$ or (ii) the loss of an ancestrally inherited genetic element $[34,36]$. We explored the origin and evolution history of the introns found in F. tricinctum and $F$. avenaceum, and searched, for each intron, its available orthologous sequences to compare their phylogenetic relationships to the one established using the compiled typical mitochondrial protein genes in close and distant Ascomycota species (Fig. 3 and Table 2). Among the nine introns characterized in the F. tricinctum and/or F. avenaceum studied strains, eight were found widely distributed in the Fusarium genus, including in members of other Fusarium species complexes. Only the intron nad5 iFtri1 was found solely in strains belonging to the FTSC, precisely in all the 14 studied F. tricinctum strains plus $2 \mathrm{~F}$. avenaceum strains out of 11 studied, suggesting that this intron is a rare intron ancestrally inherited in the FTSC. This specificity suggests that its evolution history in the Ascomycota phylum would be intron loss rather than gain and spreading.

The patchy distribution of the eight other introns in the Fusarium genus as well as their optionality in the 25 studied strains of the FTSC clearly argue in favor of their ancestral origin in the Fusarium genus, accompanied by frequent events of gain and loss occurring during short evolution periods. Among the nine different introns characterized in the $F$. tricinctum and $F$. avenaceum mitochondrial genomes, three were present in all the 25 studied strains, and the other six were found optional. Among the latter, this optionality was inter-specific for nad4L1iFave1 (present in all $F$. avenaceum strains and absent from all $F$. tricinctum strains) and cox 1 iFtri1 (present in all F. tricinctum strains and absent from all $F$. avenaceum strains). The presence/ absence of these introns thus provide a clear discrimination of $F$. tricinctum and F. avenaceum species, among the 25 studied strains. The observed high percentages of nucleotide identity between orthologous intron sequences from phylogenetically distant species supports the hypothesis of intron gains after lateral transfers. Here, up to eight lateral transfers have been evidenced and concern the introns nad4L iFave1, cob i1, cox1 iFave1, cox1 iftri1, cox1 i2, and cox1 i3 (Table 2).
In most of the species of the fungal kingdom, the mitochondrial cox 1 gene is the richest in group I introns [22], sometimes leading to astonishing "long" genes containing up to 18 introns in the Basidiomycota Agaricus bisporus. In Ascomycota, "long genes" have also been described, especially in species belonging to the Fusarium genus [18] in the FSAMSC (up to 13 introns in F. graminearum) or in the basal FSSC (eight introns reported in F. solani). On the contrary, species of the FOSC do not possess any intron in their cox1 gene [19]. These reports argue for a high mobility of the group I introns of the cox 1 gene, with frequent events of gain and loss during the evolution of the Fusarium genus. Our results support this high mobility of the cox 1 mitochondrial introns in the Fusarium genus, with evidences of lateral transfers between introns described in the FTSC and F. mangiferae (FFSC) for cox 1 iFave1, and three introns of $F$. solani (FSSC) for cox 1 iFtri1, cox 1 i2 and cox 1 i3. The co-location of these three introns in the same 3 ' region of $\operatorname{cox} 1$ and the percentages of nt identity between orthologous sequences suggest that they could have been transferred at the same time in a single event. Moreover, for cox 1 iFtri1 and $\operatorname{cox} 1$ i3, a second horizontal transfer may also have occurred involving highly distant species, an ancestor of the Basidiomycota Agrocybe agegerita and of the Ascomycota A. tubigensis, respectively. Such lateral transfers between an Ascomycota and A. aegerita has previously been reported [37]. Considering the nad4L iFave1, our results suggest two hypotheses: (i) this Fusarium ancestral mobile genetic element may have been lost (as in F. tricinctum) then recently re-gained by an event of lateral transfer involving $F$. avenaceum and a species phylogenetically related to $T$. asperellum, or (ii) the ancestral nad4L iFave1 intron may have been maintained and recently transferred to $T$. asperellum by a lateral transfer. In the absence of other Ascomycota orthologous sequence with high nucleotide identity with the $T$. asperellum intron sequence, the second hypothesis seems more likely. Similarly, the incongruent relationship between the cob il intron sequences of F. tricinctum and $F$. avenaceum strains with the orthologous sequence reported in F. venenatum suggests that a lateral transfer of this mobile genetic element may have occurred between $F$. venenatum and an ancestor of $F$. tricinctum and F. avenaceum. Notably, since cob $i 1$ is present in all $F$. avenaceum studied strains but not in all F. tricinctum ones, this intron may have been lost during the most recent evolution leading to the clade here referred as group A of the $F$. tricinctum species.

Comparing intron sequence polymorphism between $F$. tricinctum INRA104 and F. avenaceum FaLH27, little variability was detected, i.e., at levels comparable to those found in mitochondrial CDS sequences. Moreover, all the nine introns carried an intact heg encoding a 
potentially functional enzyme. Twenty years ago, Goddard and Burt [36], studying the behavior of the mitochondrial $\omega$-heg and its host intron in 20 yeast species, established a cyclic model with three steps: invasion, erosion and finally loss, followed by re-invasion. Their data led to the estimation that each step is achieved in about two million years, and that a long-term maintenance of an intact heg requires frequent lateral transmission events. This model [36] was later confirmed and extended by Koufopanou et al. [38] who reported a high adaptation of a heg to its lateral transmission. The frequency of these lateral transmission events appears, consequently, to play a major role in the widespreading of mitochondrial group I introns and heg. According to this cyclic model of intron evolution, maintenance of a functional $\mathrm{HE}$ is required only during the invasion phase of these self-splicing mobile genetic elements. When the parasitic element is fixed in a population, the HE activity becomes unnecessary, submitted to neutral selection and can be randomly lost [36, 39]. However, numerous putatively functional HEs were also reported to be maintained over long evolutionary periods in populations of species considered as mostly asexual. Such findings and implications have been extensively discussed by Gogarten et al. [39]. In this paper, they list several hypotheses to explain the maintenance of a functional $\mathrm{HE}$, such as, for example, (i) an unsuspected level of intra-specific (sexual) and/or inter-specific matings, (ii) a complex population structure where the mobile element is not fixed, or (iii) an unknown role of the HE which will be selected because increasing the fitness of its host. Here, all these hypotheses could also explain the high conservation and putative functionality observed with all the nine hegs and group I introns described in the F. tricinctum and $F$. avenaceum mitochondrial genomes, independently from the fact that these mobile genetic elements were found involved in lateral transfers (as it is the case for most of them) or not.

\section{Conclusions}

Fusarium tricinctum and Fusarium avenaceum are two phylogenetically close phytopathogenic species, producers of "emerging" mycotoxins including enniatins and, thus, potentially involved in future food-safety crises. In this paper, a comparative analysis of the molecular organization of their mitochondrial genome has been carried out in a set of 25 wild strains representing both species. This analysis shows that both species can easily be differentiated by polymorphic mitochondrial sequences, such as Indels occurring in the Lv-uORF region.

Moreover, both genomes were shown to harbour optional (inter- or intra-specifically) group I introns, all carrying putatively functional hegs, arguing for a high mobility of these introns occurring during short evolution periods. The gain events of these selfish (parasitic) mobile elements were shown to involve, for most of them, lateral transfers between phylogenetically distant species, suggesting an important role of sexual but also interspecific mating in the populations of these phytopathogens.

This study has also revealed a new type of mobile genetic element constituted by a palindromic arrangement of single nucleotide (A) $\mathrm{n}$ and (T) $\mathrm{n}$ microsatellite sequences whose presence was related to occurrence of SNPs and Indels in the neighbouring regions. Such mobile elements could represent an important driving force of mitochondrial genome evolution in Fusarium species but also in a broader range of fungal species.

\section{Methods}

Fusarium spp. strains and culture conditions, sequences used for taxonomic identification and phylogenetic studies All the F. tricinctum and F. avenaceum strains used in this study (Additional file 3: Table S3) were issued from subcultures and monosporal (conidial) isolation of strains harvested from the wild (from maize or cereal crops with various geographical origins but mostly from various French regions) and are conserved in the INRA UR1264 MycSA laboratory or in the Fungal Culture Collection of the International Centre of Microbial Resources (CIRM$\mathrm{CF}$ ), curated (Marseille, France). Mycelia were cultured on solid PDA medium (Difco). All strains were previously assigned to the $F$. tricinctum or $F$. avenaceum species by, first, a morphological characterization of the mycelial hyphae and their produced asexual (macroconidia) and/or vegetative (chlamydospores) mitospores (according to [40]), then by using, qPCR, with species-specific primers $[41,42]$. The Accession numbers of the sequences obtained in this study and used to build phylogenetic trees are also indicated in Additional file 3: Table S3.

\section{Mitochondrial genome annotation}

The complete mitochondrial genome sequences of $F$. tricinctum strain INRA104 and F. avenaceum strain FaLH27 were previously obtained $[16,17]$. Their full annotation was performed and manually curated after comparison with sequences in GenBank and EMBL databases using BLASTn and BLASTp [43]. To identify protein coding genes, MtDNA sequences were searched for open reading frames (ORF) using the ORFfinder software (https://www.ncbi.nlm. nih.gov/orffinder/). GeSeq [44] was further used for the annotation of $F$. tricinctum INRA104 and $F$. avenaceum FaLH27 guided by the mitochondrial genomes of $F$. verticillioides, F. solani, and F. graminearum as annotated in MiToFun (http://mitofun.biol.uoa.gr/index.html). Predictions were then crossed and confirmed by multi-alignments of nucleic acids and predicted protein sequences performed using Clustal Omega [45] and MUSCLE [46]. Mitochondrial transfer RNA genes (tRNAs) were located using the tRNA Scan-SE [47]. Mitochondrial maps and corresponding 
Genbank files were generated using ApE (http://jorgensen. biology.utah.edu/wayned/ape/) and SnapGene software (from GSL Biotech; available at snapgene.com). Introns were first identified by Blast analysis. For each intron, its borders ( 5 ' and $3^{\prime}$ ends) were established after deletion of the highly conserved exonic sequences of each mitochondrial CDS then confirmed by alignment with orthologous sequences. In the same way, the assignment to group I and subgroup were deduced from those given to their orthologous sequences, mainly in Al-Reedy et al. [18] or Ferandon et al. [22] for coxl introns.

\section{DNA extraction and PCR}

Whole genomic DNA was extracted according to the $N$ cethyl-NNN-trimethyl ammonium bromide (CTAB) procedure described in Barroso et al. [48].

Primers were designed to amplify and sequence nuclear and mitochondrial regions used as taxonomic and phylogenetic markers (Additional file 3: Table S4). These couples were defined from the alignment of the F. tricinctum and $F$. avenaceum nuclear gene sequences $r p b 1, r p b 2$, and of a part of the mitochondrial Lv-uORF. Primers were also designed to amplify each intron found in the mitochondrial sequences of $F$. tricinctum strain INRA104 or F. avenaceum strain FaLH27 (Additional file 3: Table S4). PCR mixes contained between 10 to $100 \mathrm{ng}$ of fungal DNA, $1 \mu \mathrm{M}$ of both primers, $100 \mu \mathrm{M}$ of each dNTP, 2 units of GoTaq DNA polymerase (Promega Corp., Madison, Wis, USA) in a final volume of $50 \mu \mathrm{L}$ buffer. PCR conditions were: $95^{\circ} \mathrm{C}$ for $5 \mathrm{~min}$; $35 \mathrm{x}\left(95^{\circ} \mathrm{C}\right.$ for $30 \mathrm{~s}$; primer hybridization at the indicated temperature (Additional file 3: Table S4) for $30 \mathrm{~s}$; elongation at $72{ }^{\circ} \mathrm{C}$ for 90 to $120 \mathrm{~s}$ ); $72^{\circ} \mathrm{C}$ for $5 \mathrm{~min}$.

\section{DNA sequencing and sequence analysis}

PCR products were sequenced, according to the Sanger method, on both strands by Genewiz (UK), with both forward and reverse primers used to obtain each PCR product.

GenBank Accession numbers of the sequenced PCR products are indicated in Additional file 3: Table S3. Sequence comparisons with GenBank and EMBL databases were performed with BLAST [43]. Multi-alignments of nucleic acids were performed with MUSCLE 3.8.31 [46] and subsequently cleaned with Gblocks 0.91b [49]. Phylogenetic trees were computed using MrBayes 3.2.7a [50, 51] and parameters (Additional file 3, Table S5) estimated with MEGA X [52]. Tree rendering was performed with FigTree 1.4.4, displaying posterior probabilities as branch supports obtained after 1,000,000 generations.

\section{Supplementary information}

Supplementary information accompanies this paper at https://doi.org/10. 1186/s12864-020-6770-2.

Additional file 1: GenBank formatted annotated sequence of the $F$. tricinctum strain INRA104 MtDNA.

Additional file 2: GenBank formatted annotated sequence of the $F$. avenaceum strain FaLH27 MtDNA.

Additional file 3: Table S1. SNP and indel occurrence in F. tricinctum vs. F. avenaceum mitochondrial genomes. Table S2. Details of SNPS and Indels between F. tricinctum vs. F. avenaceum mitochondrial genomes. Table S3. F. tricinctum and F. avenaceum strains and sequences (GenBank Accession $N^{\circ}$ ) used in this study. Table S4. Primers and PCR conditions used in this study. Table S5. MrBayes input parameters estimated with MEGA X. Figure S1. Unrooted phylogenetic tree of Fusarium species and of distant or closely related Ascomycota species based on cox 1 complete CDS sequence alignment. Posterior probabilities (Bayesian inference; 1,000,000 generations) are indicated in red. Labels show species names followed by the name and/or number of the strain and the GenBank accession number corresponding to the sequence used. Basidio = Basidiomycota; Asco = Ascomycota; FSSC = Fusarium solani Species Complex; FSAMSC = Fusarium sambucinum Species Complex; FTSC $=$ Fusarium tricinctum Species Complex; FOSC = Fusarium oxysporum Species Complex; FFSC = Fusarium fujikuroi Species Complex. Figure S2. Nucleotide alignments of the interspecific polymorphic region between $F$. tricinctum and $F$. avenaceum of the mitochondrial large variable region containing the UORF for studied F. tricinctum and $F$. avenaceum strains.

Additional file 4: Table S6. Strains and sequences (GenBank Accession $\mathrm{N}^{\circ}$ ) used in this study

\section{Abbreviations}

Fave: Fusarium avenaceum; FFSC: Fusarium fujikuroi Species Complex; FHB: Fusarium Head Blight; FOSC: Fusarium oxysporum Species Complex; FSAMSC: Fusarium sambucinum Species Complex; FSSC: Fusarium solani Species Complex; Ftri: Fusarium tricinctum; FTSC: Fusarium tricinctum Species Complex; Indel: Insertion/deletion sequence; heg: homing endonuclease gene; LSU-rDNA: Large subunit ribosomal DNA; MtDNA: Mitochondrial genome; SNP: Single Nucleotide Polymorphism; SSU-rDNA: Small subunit rDNA; uORF: unidentified Open Reading Frame

\section{Acknowledgements}

The authors thank Manon Lecouffe and Solenne Reverbel for technical assistance.

\section{Authors' contributions}

All authors contributed to the study conception and design. NP, CG, LP-G, $C D, C Z$ and $G B$ prepared the material, performed the experiments and collected the data. Data analyses were performed by NP, JG, MF-O, FR-F, JMS, CZ and GB. The first draft of the manuscript was written by NP and GB and all authors commented on previous versions of the manuscript. All authors read and approved the final manuscript.

\section{Funding}

This work was supported by grants from the National key research and development program of China (Program number 2016YFD0501207) and the Department of Microbiology of the Food Chain (MICA) of the French National Institute for Agricultural Research (INRA). CG PhD fellowship is funded by Region Nouvelle-Aquitaine and the INRAE Department SPE. These funding had no role in the design of the study, collection, analysis and interpretation of data or in writing the manuscript.

\section{Availability of data and materials}

All the sequences determined in this study have been deposited at GenBank (see text and Additional file 3 Table $\$ 3$ for Accession numbers). All the sequences used for phylogenetic analyses have been retrieved from the GenBank. Their accession numbers are listed in additional file 4, Table S6. 


\section{Ethics approval and consent to participate}

Not applicable.

\section{Consent for publication}

Not applicable.

\section{Competing interests}

The authors declare they have no competing interests.

\section{Author details}

'INRAE, MycSA, F-33882 Villenave d'Ornon, France. ${ }^{2}$ LIPM, Université de Toulouse, INRAE, CNRS, Castanet-Tolosan, France. ${ }^{3}$ Academy of National Food and Strategic Reserves Administration, Beijing, China. ${ }^{4}$ University of Bordeaux, INRAE, MycSA, F-33882 Villenave d'Ornon, France.

\section{Received: 17 February 2020 Accepted: 4 May 2020}

\section{Published online: 12 May 2020}

\section{References}

1. Beccari G, Prodi A, Tini F, Bonciarelli U, Onofri A, Oueslati S, Limayma M, Covarelli L. Changes in the Fusarium Head Blight complex of malting barley in a three-year field experiment in Italy. Toxins (Basel). 2017;9(4):E120.

2. Beccari G, Senatore MT, Tini F, Sulyok M, Covarelli L. Fungal community, Fusarium head blight complex and secondary metabolites associated with malting barley grains harvested in Umbria, Central Italy. Int J Food Microbiol. 2018:20(273):33-42.

3. Jestoi M. Emerging Fusarium-mycotoxins fusaproliferin, beauvericin, enniatins, and moniliformin: a review. Crit Rev Food Sci Nutr. 2008; 48(1):21-49.

4. Svingen $T$, Lund Hansen $N$, Taxvig C, Vinggaard AM, Jensen U, Have RP. Enniatin B and beauvericin are common in Danish cereals and show high hepatotoxicity on a high-content imaging platform. Environ Toxicol. 2017; 32(5):1658-64

5. Xu XM, Monger W, Ritieni A, Nicholson P. Effect of temperature and duration of wetness during initial infection periods on disease development, fungal biomass and mycotoxin concentrations on wheat inoculated with single, or combinations of Fusarium species. Plant Pathol. 2007:56:943-56.

6. Shi W, Tan Y, Wang S, Gardiner DM, De Saeger S, Liao Y, Wang C, Fan Y, Wang Z, Wu A. Mycotoxigenic potentials of Fusarium species in various culture matrices revealed by mycotoxin profiling. Toxins (Basel). 2016;9(1):E6.

7. Fraeyman S, Croubels S, Devreese M, Antonissen G. Emerging Fusarium and Alternaria mycotoxins: occurrence, toxicity and toxicokinetics. Toxins (Basel). 2017;9(7):E228

8. Orlando B, Grignon G, Vitry C, Kashefifard K, Valade R. Fusarium species and enniatin mycotoxins in wheat, durum wheat, triticale and barley harvested in France. Mycotoxin Res. 2019;35(4):369-80.

9. Summerell B, Leslie JF. Fifty years of Fusarium: how could nine species have ever been enough? Fungal Divers. 2011;50:135-44.

10. Saccardo M. F. avenaceum. Sylloge fungorum (Abellini). 1886:4:713

11. Saccardo M. F. tricinctum. Sylloge fungorum (Abellini). 1886;4:700.

12. O'Donnell K, Rooney AP, Proctor RH, Brown DW, McCormick SP, Ward TJ, Frandsen RJN, Lysøe E, Rehner SA, Aoki T, Robert VARG, Crous PW, Groenewald JZ, Kang S, Geiser DM. Phylogenetic analyses of RPB1 and RPB2 support a middle cretaceous origin for a clade comprising all agriculturally and medically important Fusaria. Fungal Genet Biol. 2013;52:20-31.

13. Wang H, Xiao M, Kong F, Chen S, Dou HT, Sorrell T, Li RY, Xu YC. Accurate and practical identification of 20 Fusarium species by seven-locus sequence analysis and reverse line blot hybridization, and an in vitro antifungal susceptibility study. J Clin Microbiol. 2011;49(5):1890-8.

14. Waalwijk C, Taga M, Zheng SL, Proctor RH, Vaughan MM, O'Donnell K. Karyotype evolution in Fusarium. IMA Fungus. 2018;9(1):13-26.

15. O'Donnell K, McCormick SP, Busman M, Proctor RH, Ward TJ, Doehring G, Geiser DM, Alberts JF, Rheeder JP. 2018. Marasas et al. "toxigenic Fusarium species: identity and mycotoxicology" revisited. Mycologia. 1984;110(6): 1058-80.

16. Ponts N, Richard-Forget F, Zhang H, Barroso G, Zhao C. Genome sequence of the emerging mycotoxin-producing filamentous fungus Fusarium tricinctum strain INRA104. Genome Announc. 2018;6(25):e00509-18.

17. Lysøe E, Harris LJ, Walkowiak S, Subramaniam R, Divon HH, Riiser ES, Llorens C, Gabaldón T, Kistler HC, Jonkers W, Kolseth AK, Nielsen KF, Thrane U,
Frandsen RJ. The genome of the generalist plant pathogen Fusarium avenaceum is enriched with genes involved in redox, signaling and secondary metabolism. PLoS One. 2014;9(11):e112703.

18. Al-Reedy RM, Malireddy R, Dillman CB, Kennell JC. Comparative analysis of Fusarium mitochondrial genomes reveals a highly variable region that encodes an exceptionally large open reading frame. Fungal Genet Biol. 2012;49(1):2-14

19. Brankovics B, van Dam P, Rep M, de Hoog GS, van der Lee TA J, Waalwijk C, van Diepeningen AD. Mitochondrial genomes reveal recombination in the presumed asexual Fusarium oxysporum species complex. BMC Genomics. 2017;18(1):735.

20. Aguileta G, de Vienne DM, Ross ON, Hood ME, Giraud T, Petit E, Gabaldón T. High variability of mitochondrial gene order among fungi. Genome Biol Evol. 2014;6(2):451-65.

21. Bullerwell $C E$, Burger $G$, Lang BF. A novel motif for identifying rps3 homologs in fungal mitochondrial genomes. Trends Biochem Sci. 2000; 25(8):363-5.

22. Ferandon C, Moukha S, Callac P, Benedetto JP, Castroviejo M, Barroso G. The Agaricus bisporus cox 1 gene: the longest mitochondrial gene and the largest reservoir of mitochondrial group 1 introns. PLoS One. 2010;5(11): e14048.

23. Pellenz S, Harington A, Dujon B, Wolf K, Schafer B. Characterization of the ISpom I endonuclease from fission yeast: insights into the evolution of a group I intron-encoded homing endonuclease. J Mol Evol. 2002;55(3):302-13.

24. Paquin B, Laforest MJ, Lang BF. Double-hairpin elements in the mitochondrial DNA of Allomyces: evidence for mobility. Mol Biol Evol. 2000; 17(11):1760-8

25. Tourasse NJ, Shtaida N, Khozin-Goldberg I, Boussiba S, Vallon O. The complete mitochondrial genome of the green microalga Lobosphaera (Parietochloris) incisa reveals a new type of palindromic repetitive repeat. BMC Genomics. 2015;16:580.

26. Kim Jl, Yoon HS, Yi G, Shin W, Archibald JM. Comparative mitochondrial genomics of cryptophyte algae: gene shuffling and dynamic mobile genetic elements. BMC Genomics. 2018;19:275.

27. Nargang FE, Bell JB, Stohl LL, Lambowitz AM. A family of repetitive palindromic sequences found in Neurospora mitochondrial DNA is also found in a mitochondrial plasmid DNA. J Biol Chem. 1983;258(7):4257-60.

28. Yin S, Heckman J, RajBhandary UL. Highly conserved GC-rich palindromic DNA sequences flank tRNA genes in Neurospora crassa mitochondria. Cell. 1981;26(3 Pt 1):325-32.

29. Koll F, Boulay J, Belcour L, D'Aubenton-Carafa Y. Contribution of ultra-short invasive elements to the evolution of the mitochondrial genome in the genus Podospora. Nucleic Acids Res. 1996;24(9):1734-41.

30. Wu B, Hao W. A Dynamic Mobile DNA Family in the Yeast Mitochondrial Genome. G3 (Bethesda). 2015:5(6):1273-82.

31. Kulik T, Brankovics B, Sawicki J, van Diepeningen A. The complete mitogenome of Fusarium gerlachii. Mitochondrial DNA A DNA Mapp Seq Anal. 2016;27(3):1895-6.

32. Kulik T, Brankovics B, Sawicki J, van Diepeningen A. The complete mitogenome of Fusarium culmorum. Mitochondrial DNA A DNA Mapp Seq Anal. 2016;27(4):2425-6.

33. Ferandon C, Xu JP, Barroso G. The $135 \mathrm{kbp}$ mitochondrial genome of Agaricus bisporus is the largest known eukaryotic reservoir of group I introns and plasmid-related sequences. Fungal Genet Biol. 2013;55:85-91.

34. Cusimano N, Zhang LB, Renner SS. Reevaluation of the cox 1 group I intron in Araceae and angiosperms indicates a history dominated by loss rather than horizontal transfer. Mol Biol Evol. 2008;25(2):265-76.

35. Sanchez-Puerta MV, Cho Y, Mower JP, Alverson AJ, Palmer JD. Frequent, phylogenetically local horizontal transfer of the cox 1 group I intron in flowering plant mitochondria. Mol Biol Evol. 2008;25(8):1762-77.

36. Goddard MR, Burt A. Recurrent invasion and extinction of a selfish gene. Proc Natl Acad Sci U S A. 1999;96(24):13880-5.

37. Gonzalez $P$, Barroso $G$, Labarère J. Molecular analysis of the split cox1 gene from the Basidiomycota Agrocybe aegerita: relationship of its introns with homologous Ascomycota introns and divergence levels from common ancestral copies. Gene. 1998;220(1-2):45-53.

38. Koufopanou V, Goddard MR, Burt A. Adaptation for horizontal transfer in ahoming endonuclease. Mol Biol Evol. 2002;19(3):239-46.

39. Gogarten JP, Hilario E. Inteins, introns, and homing endonucleases: recent revelations about the life cycle of parasitic genetic elements. BMC Evol Biol. 2006;6:94. 
40. Leslie JF, Summerell BA. Fusarium laboratory workshops-a recent history Mycotoxin Res. 2006;22(2):73-4.

41. Turner S, Lees AK, Rezanoor HN, Nicholson P. Refinement of PCR-detection of Fusarium avenaceum and evidence from DNA marker studies for phenetic relatedness to Fusarium tricinctum. Plant Pathol. 1998:47:278-88.

42. Sonia E, Siou D, Gélisse S, Cruaud C, Lannou C, Lebrun MH, Laval V. Optimized real time QPCR assays for detection and quantification of Fusarium and Microdochium species involved in wheat head blight as defined by MIQE guidelines. bioRxiv. 2018. https://doi.org/10.1101/272534.

43. Altschul SF, Gish W, Miller W, Myers EW, Lipman DJ. Basic local alignment search tool. J Mol Biol. 1990;215(3):403-10.

44. Tillich M, Lehwark P, Pellizzer T, Ulbricht-Jones ES, Fischer A, Bock R, Greiner S. GeSeq - versatile and accurate annotation of organelle genomes. Nucleic Acids Res. 2017;45:W6-W11.

45. Sievers F, Wilm A, Dineen D, Gibson TJ, Karplus K, Li W, Lopez R, McWilliam H, Remmert M, Söding J, Thompson JD, Higgins DG. Fast, scalable generation of high-quality protein multiple sequence alignments using Clustal Omega. Mol Syst Biol. 2011;11(7):539.

46. Edgar RC. MUSCLE: multiple sequence alignment with high accuracy and high throughput. Nucleic Acids Res. 2004;32(5):1792-7.

47. Lowe TM, Eddy SR. tRNAscan-SE: a program for improved detection of transfer RNA genes in genomic sequence. Nucleic Acids Res. 1997;25(5): $955-64$

48. Barroso G, Blesa S, Labarere J. Wide distribution of mitochondrial genome rearrangements in wild strains of the cultivated Basidiomycete Agrocybe aegerita. Appl Environ Microbiol. 1995;61:1187-93.

49. Castresana J. Selection of conserved blocks from multiple alignments for their use in phylogenetic analysis. Mol Biol Evol. 2000;17:540-52.

50. Huelsenbeck JP, Ronquist F. MRBAYES: Bayesian inference of phylogenetic trees. Bioinformatics. 2001;17:754-5.

51. Ronquist F, Huelsenbeck JP. MrBayes 3: Bayesian phylogenetic inference under mixed models. Bioinformatics. 2003;19:1572-4.

52. Kumar S, Stecher G, Li M, Knyaz C, Tamura K. MEGA X: Molecular evolutionary genetics analysis across computing platforms. Mol Biol Evol. 2018:35:1547-9.

\section{Publisher's Note}

Springer Nature remains neutral with regard to jurisdictional claims in published maps and institutional affiliations.

Ready to submit your research? Choose BMC and benefit from:

- fast, convenient online submission

- thorough peer review by experienced researchers in your field

- rapid publication on acceptance

- support for research data, including large and complex data types

- gold Open Access which fosters wider collaboration and increased citations

- maximum visibility for your research: over $100 \mathrm{M}$ website views per year

At $\mathrm{BMC}$, research is always in progress.

Learn more biomedcentral.com/submissions 